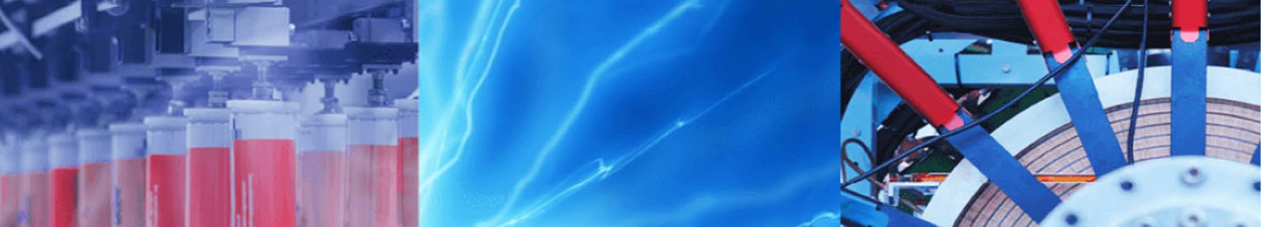

Research Article

\title{
Radium in groundwater hosted in porous aquifers: estimation of retardation factor and recoil rate constant by using NAPLs
}

\author{
Alessandra Briganti ${ }^{1} \cdot$ Mario Voltaggio $^{2} \mathbb{D} \cdot$ Paola Tuccimei $^{1} \cdot$ Michele Soligo $^{1}$
}

Received: 17 February 2020 / Accepted: 29 September 2020 / Published online: 1 November 2020

(c) Springer Nature Switzerland AG 2020

\begin{abstract}
A new method for estimating retardation factor and recoil constant of radium isotopes in groundwater hosted in porous aquifers is described. The method is based on the evidence that alpha-recoiled radium ions, supplied by thorium parent atoms which occur in phases immersed in NAPL (Non-Aqueous Phase Liquids), are not adsorbed on solid phases. Experimental evidence is given that manganese dioxide, zeolite 4A, natural clay, monazite and weathered volcanic rock, all phases normally adsorbing radium from aqueous solutions, when immersed in NAPL adsorb negligible amounts of radium. This allows using experimental data on rock samples, representative of porous aquifers, for estimating Ra retardation factor and its alpha recoil constant in groundwater, without using $\mathrm{Rn}$ data as a comparison term. Unlike estimation of retardation factor between the "NAPL method" and the method based on comparison with radon depends on the different process of entry from aquifer rock into groundwater for radon and radium. Precise estimates of retardation factor and recoil constants of radium allow to apply equations ruling the temporal evolution of radium isotopes in groundwater and to determine its age. Implications, useful for measuring the contamination age of soils by NAPL fluids, are described as well.
\end{abstract}

Keywords Retardation factor $\cdot$ Recoil constant $\cdot$ Radium isotopes $\cdot$ NAPL $\cdot$ Groundwater

\section{Introduction}

The goal of this paper is to provide new insights for determining groundwater ages using radium isotopes. When using radioactive tracers, the tracer age differs from the groundwater age by a factor, which is named tracer retardation factor. This factor, in case of conservative tracers, equals the unity. It should be noted that the groundwater age remains only an estimate of the mean residence time (MRT) of groundwater in aquifer [1]. In fact the shape of the age distribution in real-world groundwater is unknown. Thus, MRT is a weighted average over many idealized ages and the age of a single sample of groundwater can differ notably from the MRT of groundwater.

$\mathrm{Ra}$ isotopes are a quartet of radioactive isotopes with half lives extending from some days up to thousands of years $\left({ }^{224} \mathrm{Ra} t_{1 / 2}=3.66\right.$ days, ${ }^{223} \mathrm{Ra} t_{1 / 2}=11.4$ days, ${ }^{228} \mathrm{Ra}$ $t_{1 / 2}=5.75$ years, and ${ }^{226} \mathrm{Ra} t_{1 / 2}=1600$ years). They belong to the natural radioactive series of ${ }^{238} \mathrm{U}\left({ }^{226} \mathrm{Ra}\right),{ }^{235} \mathrm{U}\left({ }^{223} \mathrm{Ra}\right)$ and ${ }^{232} \mathrm{Th}\left({ }^{224} \mathrm{Ra}\right.$ and $\left.{ }^{228} \mathrm{Ra}\right)$. The hydrogeochemistry of radium depends mainly on four factors [2]: (1) a low ionic potential that causes strong adsorption of $\mathrm{Ra}$ ions on the

Electronic supplementary material The online version of this article (https://doi.org/10.1007/s42452-020-03610-4) contains supplementary material, which is available to authorized users.

$\triangle$ Mario Voltaggio, voltaggiomario@gmail.com; mario.voltaggio@igag.cnr.it; Alessandra Briganti, alessandra.briganti2@uniroma3.it; Paola Tuccimei, paola.tuccimei@uniroma3.it; Michele Soligo, michele.soligo@uniroma3.it |' ${ }^{1}$ Science Department, Università Roma TRE, viale G. Marconi, 00146 Rome, Italy. ${ }^{2}$ CNR-IGAG (Institute of Environmental Geology and Geoengineering), Area della Ricerca di Roma 1, via Salaria km 29, 300, 00146 Rome, Italy. 
surface of aquifer rocks; (2) coprecipitation with barium in sulphate and carbonatic groundwaters; (3) mobilization from aquifer rocks by chemical processes like dissolution of Ra-hosting minerals or Ra desorption from aquifer rocks in saline groundwaters; (4) mobilization from aquifer rocks in groundwater by the physical processes of alpha-recoil.

The temporal evolution of Ra isotopes in groundwater hosted in porous aquifers, when secular equilibrium in host rocks is assumed and Ra precipitation rate can be neglected, is given by the following master equation, whose derivation is given in Appendix 1: [2-7]: product of the constant decay $\lambda_{i}$ of ${ }^{i}$ Ra and $f_{\mathrm{a}_{1}}$ the fraction of ${ }^{i} \mathrm{Ra}_{\mathrm{r}}$ lost by a-recoil [11]:

$\Gamma_{i}=\lambda_{i} \cdot f_{\alpha}$

\subsubsection{Alpha-recoil constant}

$\mathrm{Rn}$ isotopes supply to groundwater, according Krishnawami [10], is considered as a measure of the supply of Ra isotopes as well. In this paper, this approach will be questioned. We will provide experimental evidence that

${ }^{i} \operatorname{Ra}_{w}=\left[\frac{\left(\Delta_{i}+\Gamma_{i}\right)}{\left(\lambda_{i} \cdot \mathrm{R}_{i}\right)}\right] \cdot\left(1-\mathrm{e}^{-\lambda_{i} \cdot R_{i} \cdot T}\right) \cdot\left({ }^{i} \operatorname{Ra}_{r} \cdot \frac{\rho_{r}}{\Phi}\right)+{ }^{i} \operatorname{Ra} a_{w(0)} \cdot\left(\mathrm{e}^{-\lambda_{i} \cdot R_{i} \cdot T}\right)$

where, for a Ra isotope $i,{ }^{i} \mathrm{Ra}_{w}$ is its activity in water $\left(\mathrm{Bq} \cdot \mathrm{L}^{-1}\right)$ at time $T$ (a) (i.e. groundwater age), ${ }^{i} \mathrm{Ra}_{w(0)}$ its initial activity in water $\left(\mathrm{Bq} \cdot \mathrm{L}^{-1}\right){ }_{,}^{i} \mathrm{Ra}_{r}$ the activity in the aquifer rock $\left(\mathrm{Bq} \cdot \mathrm{kg}^{-1}\right), \mathrm{R}_{i}$ its retardation factor (dimensionless), $\lambda_{i}\left(\mathrm{a}^{-1}\right)$ its decay constant, $\Delta_{i}$ its dissolution rate constant $\left(a^{-1}\right)$, $\Gamma_{i}$ its a-recoil rate constant $\left(a^{-1}\right)$ and finally $\Phi$ and $\rho_{r}$ the porosity and the bulk density of the aquifer rock.

If ${ }^{i} R a_{w(0)}$ can be neglected, Eq. 1 reduces to:

${ }^{i} \mathrm{Ra}_{w}=\left[\frac{\left(\Delta_{i}+\Gamma_{i}\right)}{\left(\lambda_{i} \cdot \mathrm{R}_{i}\right)}\right] \cdot\left(1-\mathrm{e}^{-\lambda_{i} \cdot \mathrm{R}_{i} \cdot T}\right) \cdot\left({ }^{i} \mathrm{Ra}_{r} \cdot \frac{\rho_{r}}{\Phi}\right)$

If information about $\Delta_{i}, \Gamma_{i}, \mathrm{R}_{i}{ }^{i}{ }^{i} \mathrm{Ra}_{r} \Phi$ and $\rho_{r}$ is provided, the measurement of $R a$ isotopes in groundwater gives an estimate of the age T of groundwater. Some methods, either in the field or in laboratory, partially provide this information $[2,4,5,8]$. Here we focus on a new experimental tool for determining $\Gamma_{i}$. and $\mathrm{R}_{i}$.

\subsection{Retardation factor and a-recoil rate constant}

In aquifers, and especially in those hosted in porous rocks, sorption reactions cause partitioning of reactive solute onto the solid phases composing the porous rocks. Sorption retards the transport of reactive solutes (as radium ions) making their average velocity over the travel path less than that of the groundwater flow, which is generally assumed equal to that of nonreactive/conservative solutes (as radon atoms). In the case of linear adsorption reactions [9], the ratio of nonreactive solute velocity to reactive solute velocity, which corresponds to the inverse of the ratio of their MRTs, is called retardation factor, R. Aquifer rock supplies Rn predominantly by a-recoil and this supply is orders of magnitude higher than the contribution from in situ Ra decay or rock dissolution [10]. The a-recoil rate constant $\Gamma_{i}$ of ${ }^{i} \mathrm{Ra}$, or recoil ejection rate of ${ }^{i} \mathrm{Ra}$ [5], which has the dimension of the inverse of a time, is defined as the the modality of Ra supply to groundwater differs notably from that of Rn. Consequently, not only $R_{i}$ but also the alpha recoil constant $\Gamma_{i}$ of ${ }^{i}$ Ra in groundwater should be reconsidered, having been overestimated. In this frame, it is useful to consider the Rn emanation from solid materials into air for understanding the peculiar behaviour of $\mathrm{Rn}$ in comparison with Ra in groundwater [12]. A convincing analysis of the production of $\mathrm{Rn}$ is given by Maraziotis [13] and it can be applied to any other a-recoiled radionuclide like $\mathrm{Ra}$ as well. The Rn emanation coefficient, $f$, has three components: $f_{\mathrm{r}}$, direct recoil; $f_{\mathrm{p}}$, intraparticle pore recoil; $f_{\mathrm{d}^{\prime}}$, solid-state diffusion. The direct recoil component, $f_{\mathrm{r}^{\prime}}$ is the fraction of radon escaping from a particle due to alpha-recoil processes which follow the radium decays in the outer nanometric thickness of the particle. The intraparticle pore recoil component, $f_{p^{\prime}}$ is the fraction of radon that enters the nanopore framework (i.e.nanocrack framework) of the particle due to alpha-recoil processes from radium decays inside the particle. Finally, the solid-state diffusion component, $f_{\mathrm{d}}$, is the fraction of radon that exits a solid particle due to its diffusion within definite (crystalline) or non-definite (amorphous) lattice of the particle. The definition of these components is given by Eqs. 35-37 (see Appendix 2). For ${ }^{220} \mathrm{Rn}$ (Fig. 1), due to its short halflife, the situation is simpler, since $f_{\mathrm{d}}$ is negligible at ambient temperature [14]. These components depend only on variables linked to the particle from what Rn emanates, so Eqs. 35-37 apply to Ra emanation from solids into water as well. Let us to consider now the Rn and Ra emanation into groundwater in saturated aquifers. The diffusive component $f_{\mathrm{d}}$ is considered null for Ra emanation (see Appendix 3). Thus, if nanopores were quite water-filled and $\mathrm{Rn}$ and Ra were produced only by a-recoil $\left(f_{\mathrm{d}}=0\right)$, then the $R n / R a$ ratio in groundwater should represent actually $R_{R a^{\prime}}$ assuming steady state conditions and secular radioactive equilibrium (i.e. that exponential term in Eq. 1: $\mathrm{e}^{-\lambda_{i} \cdot \mathrm{R}_{i} \cdot \mathrm{T}} \approx 0$ ). Nevertheless, if confined air occurs, it interrupts the water 

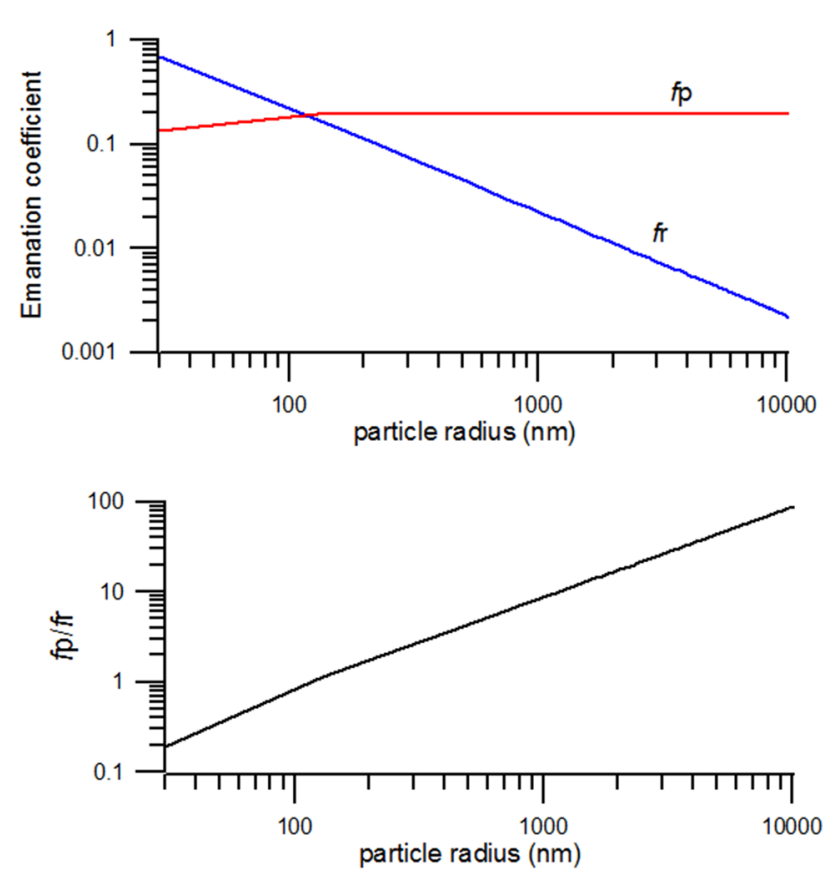

Fig. 1 Main components ( $f_{\mathrm{p}}=$ intraparticle pore recoil, $f_{\mathrm{r}}=$ direct recoil) of ${ }^{220} \mathrm{Rn}$ emanation coefficient vs. particle radius, according the model of Maraziotis [13]. Bottom: ratio between the main components vs. particle radius. Assigned conditions (Eq. 35-37): $\mathrm{k}=0.30, \mathrm{r}_{\mathrm{p}} 30 \mathrm{~nm}, \mathrm{R}_{\mathrm{c}}=30 \mathrm{~nm}$

continuity and a-recoiled Ra ions, unlike neutral $\mathrm{Rn}$ atoms, fastly attach to the walls of nanopores $[15,16]$ (Fig. 2a). In a porous medium, $f_{\mathrm{a}}$ is the sum of $f_{\mathrm{r}}$ and $f_{\mathrm{p}}$, provided that diffusion and implantation effects in adjacent grains can be neglected. Because of the presence of air in nanopores, intraparticle pore recoil component in general can be neglected as well, then $f_{\mathrm{a}}=f_{\mathrm{r}}$. Although the presence of nanopores as intermediary channels for Rn transport has been questioned $[17,18]$, other two factors advice against the use of $\mathrm{Rn}$ as reference element for a-recoil of Ra. The first one is the possible different distribution of Th and $\mathrm{Ra}$ isotopes in the particle grains of an aquifer $[19,20]$. Let us consider, for example, the ${ }^{238} \mathrm{U}$ radioactive series. Insoluble ${ }^{230} \mathrm{Th}$, parent of soluble ${ }^{226} \mathrm{Ra}$, could be mainly localized within particle grains of aquifer and within newly formed insoluble Th-phases occurring as coatings on grains [21]. Thus, ${ }^{226}$ Ra emanation depends mainly on size of particle grain or on thickness of the newly formed coatings, at least when they are in the micronic range [22]. Differently, soluble ${ }^{226} \mathrm{Ra}$, parent of ${ }^{222} \mathrm{Rn}$, due to its prolonged adsorption from groundwater, is localized, more frequently than ${ }^{230} \mathrm{Th}$, on the surface of the particle grain (Fig. 2b). In this case, the dependence of ${ }^{222} \mathrm{Rn}$ emanation on size of particle grain or on coating thickness still holds (due to the dependence of surface area of the grain) but it is different from that of ${ }^{226} \mathrm{Ra}$.

The second factor, called "back diffusion," predicts that a-recoils damage the ordered structures of crystalline phases $[11,23]$. Diffusion in radiation damage is in general faster than in ordered structures. Since Rn solid diffusion coefficient (Fig. 2c) is several orders higher than that of Ra (see Appendix 3), a further initial heavy bias is introduced when $\mathrm{Ra}$ retardation factor is calculated by comparison with $\mathrm{Rn}$ emanation. Some models include the effect of embedding (implantation of recoiled atom in the solid)
Fig. 2 Different models of radon and radium production (applied to ${ }^{238} \mathrm{U}$ series). A: ${ }^{226} \mathrm{Ra}$ and ${ }^{222} \mathrm{Rn}$ production in nanopores. $\mathrm{B}:{ }^{226} \mathrm{Ra}$ and ${ }^{222} \mathrm{Rn}$ production by direct recoil: ${ }^{226} \mathrm{Ra}$ is distributed differently from ${ }^{230} \mathrm{Th}$ covering also the grain surface. C: "back diffusion" of ${ }^{222} \mathrm{Rn}$ inside the damage

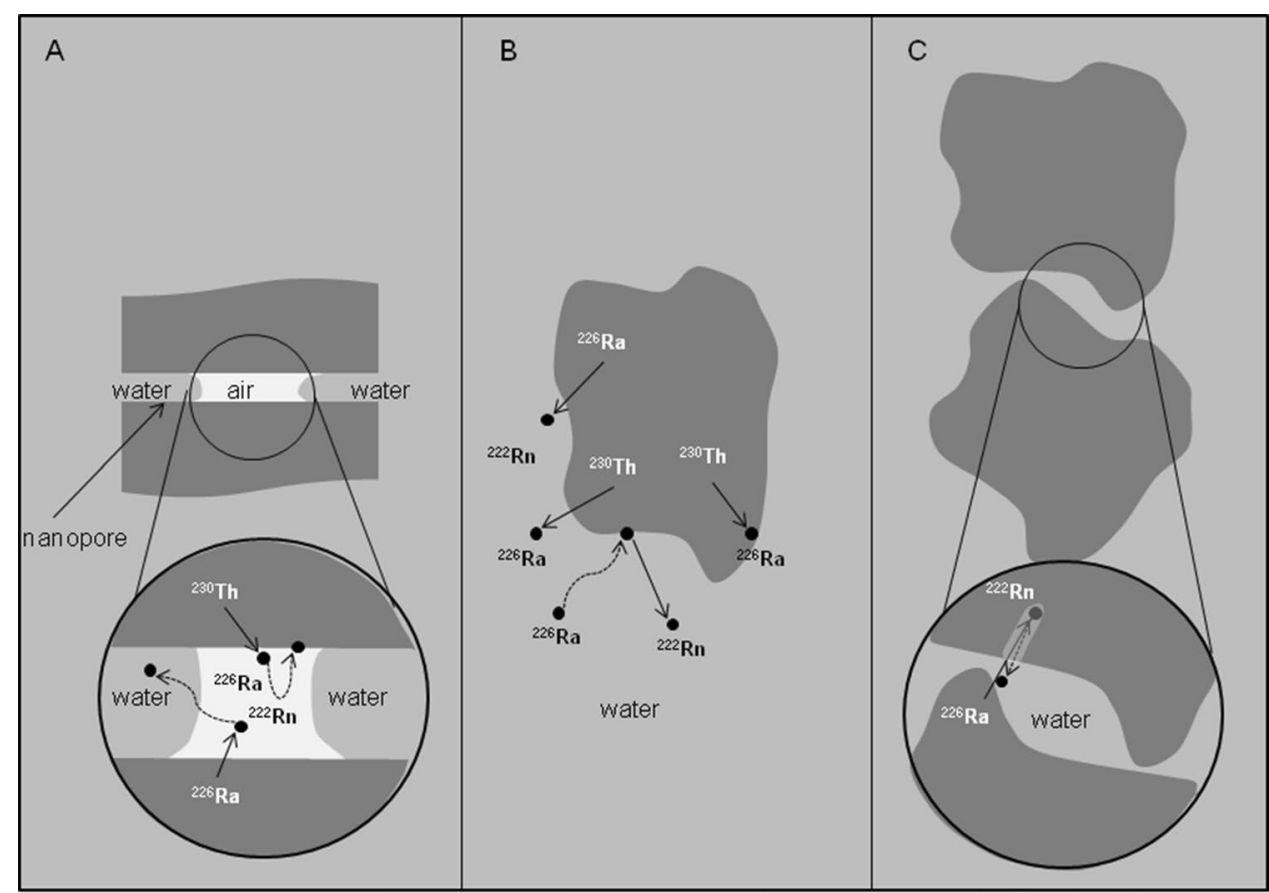

SN Applied Sciences A SPRINGER NATURE journal 
inside a nanopore as well $[22,24]$. Others studies take in account the implantation in adjacent grains, recognizing its importance for Rn emanation in water-saturated porous media $[25,26]$. In general, when parent nuclide is distributed on grain surface, the fraction $f_{\alpha}$ of a-recoiled radionuclides, topping in pore space but not implanting in adjacent grains, is defined by:

$f_{\alpha}=\frac{w}{2 \cdot R_{F}}$

where $w$ is the pore size between grains and $\mathrm{R}_{F}$ is the range of recoil $(\mathrm{nm})$ in the fluid $[26,27]$. Thus, in a watersaturated medium, when Ra is distributed at the surface of submicron particles, the relationship between Rn emanation and particle diameter is no longer inverse, due to implantation effects. Actually, any attempt of modelling the $R n$ and Ra release by a-recoil effects in an unitary scheme is complicated and ultimately unrealistic. In our opinion, it is necessary to resort to experimental tools for establishing the real $\Gamma_{l}$ and $\mathrm{R}_{i}$. This is important both for the challenge of utilizing Ra isotopes for groundwater dating [28-30] and for the problem of radionuclide transport in groundwater because of possible release of nuclear waste products into aquifers [10].

\subsubsection{Retardation factor}

The retardation factor is connected to the dimensionless adsorption distribution coefficient $K_{d}^{*}$ by:

$R=\mathrm{K}_{d}^{*}+1$

$\mathrm{K}_{d}^{*}$ is related to the dimensional adsorption distribution coefficient $\mathrm{K}_{d}\left(\mathrm{~L} \cdot \mathrm{kg}^{-1}\right)$ by [33]:

$\mathrm{K}_{d} \cdot \frac{\rho_{r}}{\Phi}=\mathrm{K}_{d}^{*}$

In fresh waters, radium ion $\left(\mathrm{Ra}^{2+}\right)$ approximates a conservative behaviour in fracturated aquifers as carbonatic or karst aquifers $[28,29]$ or in some volcanic aquifers where Ra-adsorbing mineral phases are scarce [31]. Generally, in fresh waters hosted in common fracturated crystalline rocks and in porous rock, Ra is strongly adsorbed [32], displaying an increasing retardation factor $\left(R_{R a}>>1\right)$ with decreasing grain size [3]. Ra desorption and consequent decrease in retardation factor has been observed under conditions of: (i) increasing salinity due to processes of cation competition and displacement [33, 34], (ii) decreasing mineral surface charge and (iii) increasing stability of inorganic complexes [35]. In coastal aquifers and even in marine environment $\mathrm{Ra}$ adsorption is still appreciable with the exception of hypersaline environments where radium can be considered conservative $\left(R_{R_{a}} \sim 1\right)$ [7].
According to Krishnawami, at steady-state conditions and when the term $\mathrm{e}^{-\lambda_{i} \cdot R_{i} \cdot T}$ in Eq. 1 trends to 0 (i.e. $\lambda_{i} \cdot T \approx 5$ ), retardation factors of ${ }^{226} \mathrm{Ra},{ }^{228} \mathrm{Ra}$ and ${ }^{224} \mathrm{Ra}$ are equal to the ${ }^{222} \mathrm{Rn} /{ }^{226} \mathrm{Ra},{ }^{220} \mathrm{Rn} /{ }^{228} \mathrm{Ra}$ and ${ }^{220} \mathrm{Rn} /{ }^{224} \mathrm{Ra}$ activity ratios in groundwater, respectively $[10,36]$. In terms of kinetics, the general equation for $\mathrm{R}_{i}$ of radioactive solutes is defined by $[5,10]$ :

$\mathrm{R}_{i}=1+\left[\frac{\mathrm{k}_{\mathrm{s}}}{\left(\mathrm{k}_{d}+\lambda_{i}\right)}\right]$

where $\mathrm{k}_{s}$ and $\mathrm{k}_{d}$ are the sorption and desorption rate constants $\left(t^{-1}\right)$ of the radioactive solute $i$, respectively. This equation trends to the known equation for not radioactive solutes when $\lambda_{i}$ trends to zero or when $\lambda_{i}<<k_{d}$ :

$\mathrm{R}_{i}=1+\left(\frac{\mathrm{k}_{s}}{\mathrm{k}_{d}}\right)=1+\mathrm{K}_{d}^{*}$

Returning to Eq. 2, for Ra isotopes of short mean life $\left({ }^{223} \mathrm{Ra},{ }^{224} \mathrm{Ra}\right)$ both dissolution constant and exponential term approximate to zero in few days [2]. Therefore, in the case of ${ }^{224} \mathrm{Ra}$ in groundwater, it is realistic to admit that:

${ }^{224} \mathrm{Ra}_{w}=\left(\frac{\Gamma_{224}}{\lambda_{224} \cdot \mathrm{R}_{\mathrm{Ra}-224}}\right) \cdot\left({ }^{224} \mathrm{Ra}_{r} \cdot \frac{\rho_{r}}{\Phi}\right)$

Combining Eq. 9 with Eq. 3:

$\mathrm{R}_{\mathrm{Ra}-224}=\left(\frac{f_{\alpha 224}}{{ }^{224} \mathrm{Ra}_{w}}\right) \cdot\left({ }^{224} \mathrm{Ra}_{r} \cdot \frac{\rho_{r}}{\Phi}\right)$

Equations 7-10 are valid for groundwater in moderateto-low salinity conditions and near neutral $\mathrm{pH}$.

Equation 10 is alternative to Eq. 7 for estimating $\mathrm{R}_{\mathrm{Ra}-224}$. As for $\mathrm{R}_{\mathrm{Ra}-228}$ and $\mathrm{R}_{\mathrm{Ra}-226}$, being $\lambda_{i}<<\mathrm{k}_{d}$, which is generally the case for ${ }^{228} \mathrm{Ra}$ and ${ }^{226} \mathrm{Ra}$ in groundwaters, $[10,29,37]$ :

$\mathrm{R}_{\mathrm{Ra}-228} \approx \mathrm{R}_{\mathrm{Ra}-226} \approx 1+\left[\frac{\mathrm{k}_{s}}{\mathrm{k}_{d}}\right] \neq \mathrm{R}_{\mathrm{Ra}-224}=1+\left[\frac{\mathrm{k}_{\mathrm{s}}}{\left(\mathrm{k}_{d}+\lambda_{224}\right)}\right]$

\section{Estimation of retardation factor and recoil rate constant by "NAPL method"}

Equations like Eqs. 7 and 10 are valid when the system is at a steady state and at radioactive equilibrium relatively to a particular ${ }^{i} \mathrm{Ra}$. In this case, the sum of the production and desorption rate equals the sum of decay and adsorption rate [5]. For shallow aquifers, this condition is generally satisfied for Ra isotopes of shortest life $\left({ }^{223} \mathrm{Ra},{ }^{224} \mathrm{Ra}\right)$. Usually the Ra production rate in water is monitored by $\mathrm{Rn}$ which, however, has an emanation efficiency different from Ra. 
This difficulty can be avoided, in case of porous aquifers, by using the "NAPL method." It consists in immersing two representative samples of aquifer rock in water and in a "non-aqueous phase liquid" (NAPL) and, after a comparison of measured Ra activities, in calculating the real $f_{\mathrm{a}}$. This approach assumes that adsorption of inorganic ions on mineral grains in NAPLs is negligible. The most complete study on this topic is the one of Wainipee and coworkers [38]. They have shown as NAPLs coating reduces As (V) adsorption by decreasing the available surface area of clay minerals. In fact, polar organic compounds (as negatively charged carboxylic, naphthenic acids and positively charged amines, cationic surfactants, pyridines) contained in NAPLs, easily adsorb onto the mineral surfaces. Clay edges and asperities represent pinning points for NAPLs, being negatively or positively charged. Polar components of NAPLs with opposite charges, adsorbed onto the surface of the aquifer rock, provide surfaces of low interaction energy $[39,40]$. In this way, saturation of adsorption sites and low mobility of the adsorbed organic polar compounds resemble what occurs in hypersaline aquifers where $\mathrm{R}_{i}$ trends to unity [6]. For a porous rock immersed in a non-polar liquid like NAPL, dissolution does not occur and, if adsorption is negligible, then $\mathrm{R}_{i}=1$. In such a system, after a time of 20 days (required for reaching radioactive equilibrium between ${ }^{224} \mathrm{Ra}$ and ${ }^{228} \mathrm{Th}$ ), Eq. 10 can be rewritten:

$1=\left(\frac{f_{\alpha 224}}{{ }^{224} \mathrm{Ra}_{\mathrm{NAPL}}}\right) \cdot\left({ }^{224} \mathrm{Ra}_{r} \cdot \frac{\rho_{r}}{\Phi}\right)$

where ${ }^{224} \mathrm{Ra}_{\text {NAPL }}\left(\mathrm{Bq} \cdot \mathrm{L}^{-1}\right)$ is the ${ }^{224} \mathrm{Ra}$ activity measured in NAPL; therefore:

${ }^{224} \mathrm{Ra}_{\mathrm{NAPL}}=f_{\alpha 224} \cdot\left({ }^{224} \mathrm{Ra}_{r} \cdot \frac{\rho_{r}}{\Phi}\right)$

Experimentally, $f_{\mathrm{a} 224}$ is found by:

$f_{\alpha 224}=\frac{{ }^{224} \mathrm{Ra}_{\mathrm{NAPL}} \cdot \mathrm{L} \text { of NAPL in immersion test }}{{ }^{224} \mathrm{Ra}_{\mathrm{r}} \cdot \mathrm{kg} \text { of rock in immersion test }}$

Then $f_{\mathrm{a} 224}$, through Eq. 3, gives the value of $\Gamma_{224}$.

By an analogue immersion test of 20 days, using water instead of NAPL, ${ }^{224} \mathrm{Ra}_{\mathrm{w}}$ can be measured for values of $\rho_{\mathrm{r}}$ and $\Phi$ equal to those of NAPL immersion. Thus, if all ${ }^{224} \mathrm{Ra}$ which recoils from solid to fluid during the immersion test, is desorbed from solid surface to NAPL (i.e. The dimensionless distribution coefficient of ${ }^{224}$ Ra between solid and NAPL is equal to 0$), R_{\text {Ra-224 }}$ can be easily determined by substituting Eq. 13 in Eq. 10:
$R_{R a-224}=\frac{{ }^{224} \mathrm{Ra}_{\mathrm{NAPL}}}{{ }^{224} \mathrm{Ra}_{w}}$

In this paper, the experimental way to estimate $\mathrm{R}_{\mathrm{Ra}-224}$ and $\Gamma_{224}$ by using Eqs. 13-15 will be called "NAPL method". Figure 3 shows the standard procedure for obtaining $\mathrm{R}_{i}$ and $\Gamma_{i}$ of $\mathrm{Ra}$ isotopes from representative samples of porous aquifer rocks. The essential for reproducibility of the method is that the duration of the immersion and the ratio between the weight of sample and the volume of liquid be the same in the two immersion tests (NAPL and water). Although $\mathrm{R}_{\mathrm{Ra}-224}$ and $\mathrm{r}_{224}$ can be estimated also theoretically, their determination in laboratory improves the application of Ra isotopes to groundwater dating.

Now, how to find the retardation factor for ${ }^{228} \mathrm{Ra}$ and ${ }^{226} \mathrm{Ra}$ ? Theoretically, the $\mathrm{R}_{228} / \mathrm{R}_{224}$ ratio varies between 1 and the $\lambda_{224} / \lambda_{228}$ ratio ( 273) [10]. In the real world, this range could be narrower, between 1 and 3 , as shown in Appendix 4.

Let us consider now the values of $\mathrm{R}_{\mathrm{Ra}-228}$ and $\mathrm{R}_{\mathrm{Ra}-224}$ calculated from values of $k_{d}$ and $k_{s}$ available in the literature and obtained by the Krishnawami method ( 25 groundwaters of 12 different aquifers, Fig. 4). These data can be used to estimate the $R_{\text {Ra-228 }} / R_{\text {Ra-224 }}$ ratio. When plotted together, the best fitting through origin gives a straight line whose slope, equal to the $R_{\text {Ra-228 }} / R_{\text {Ra-224 }}$ ratio, is $1.75 \pm 0.21$. $R_{\text {Ra-228 }}$ and $\mathrm{R}_{\mathrm{Ra}-226}$ therefore are estimated to about 1.75 times $\mathrm{R}_{\mathrm{Ra}-224}$. It should seem strange that data derived from a questioned method obtain a valid relationship between the $R_{i} s$ of radium. However, if absolute values of $R_{i} s$ depend strictly on the used method, it is not so for their ratio. Any $R_{i} i n$ fact is equal to the ratio of the production rate (monitored by $\mathrm{Rn}$ ) and the expected content of the Ra isotope $i$ at secular equilibrium [41]. Therefore, when $\mathrm{R}_{i} \mathrm{~s}$ are divided each other, eventual biases of the production rates, measured using Rn, cancel together.

Alternatively, the NAPL method can be also applied directly to ${ }^{228} \mathrm{Ra}$, by immersing two samples representative of aquifer rock in NAPL and in water for a time sufficient to detect a-recoiled ${ }^{228} \mathrm{Ra}$ (some months to 2 years, depending on analytical sensitivity). In this case ${ }^{228}$ Ra does not achieve the radioactive equilibrium conditions and $\mathrm{R}_{\mathrm{Ra}-228}$ is easily obtained by solving (e.g. graphically) the following nonlinear equation, where ${ }^{228} \mathrm{Ra}_{\mathrm{NAPL}}, \mathrm{R}_{\mathrm{Ra}-228}, \lambda_{228}$ and $T$ are known:

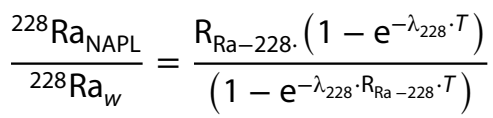



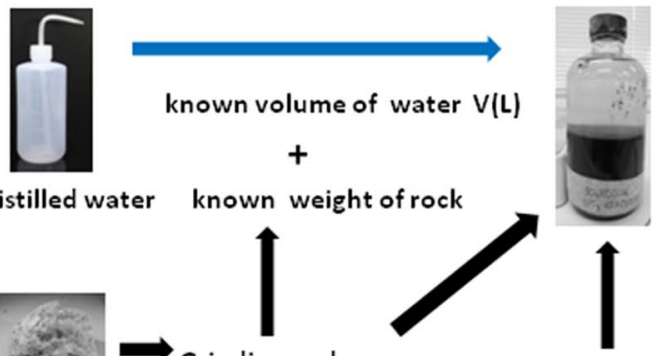

Porosity $=\Phi=V($ L $) /$ total volume Bulk density $=\rho_{r}=(1-\Phi) \cdot \rho_{d}$

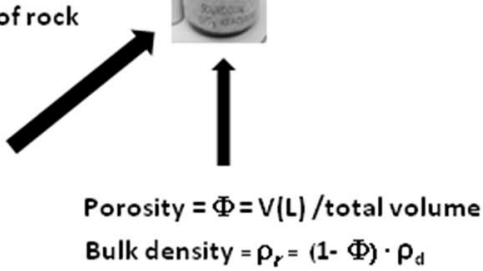

Agitation for 20 days
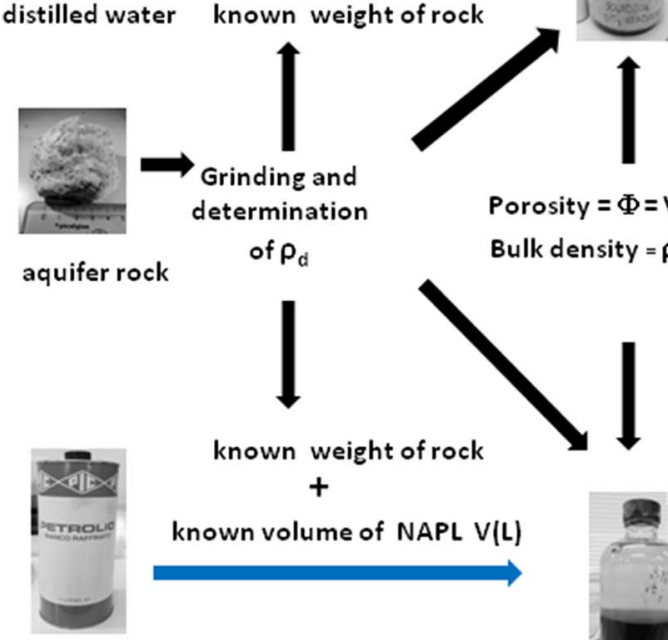
known weight of rock $+$

known volume of NAPL V(L)

Kerosene (NAPL) of $\rho_{d}$
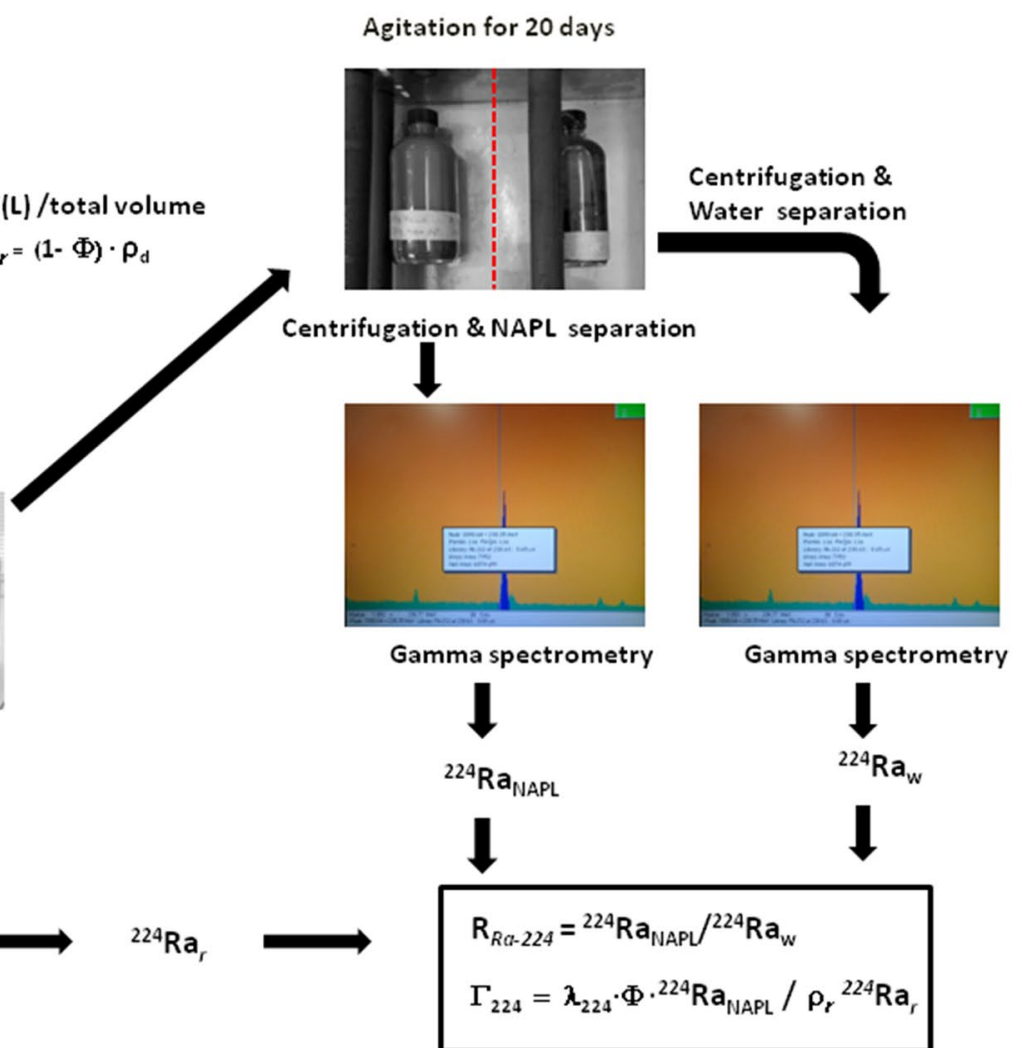

Fig. 3 Standard procedure for determining $\mathrm{R}_{\mathrm{Ra}-224}$ and $\mathrm{\Gamma}_{224}$ of radium by NAPL method

\section{Study site and application to groundwater dating by Ra isotopes: Red Pozzolane aquifer (Alban Hills Volcano, Rome)}

and by dividing Eqs. 17-18 each other and remembering that $\mathrm{R}_{R a-228}=\mathrm{R}_{R a-226}$, it follows:

The equations describing the temporal evolution of ${ }^{228} \mathrm{Ra}_{w}$ and ${ }^{226} \mathrm{Ra}_{w}$ (Eq. 1 for $i=226,228$ ) can be expressed as:

$$
\begin{aligned}
& {\left[{ }^{228} \mathrm{Ra}_{w}-\left({ }^{228} \mathrm{Ra}_{w(0)} \cdot \mathrm{e}^{-\lambda_{228} \cdot \mathrm{R}_{\mathrm{Ra}-228} \cdot T}\right)\right]=\left[\frac{\left(\Delta_{228}+\Gamma_{228}\right)}{\left(\lambda_{228} \cdot \mathrm{R}_{\mathrm{Ra}-228}\right)}\right] \cdot\left(1-\mathrm{e}^{-\lambda_{228} \cdot \mathrm{R}_{\mathrm{Ra}-228} \cdot T}\right) \cdot\left({ }^{228} \mathrm{Ra}_{r} \cdot \frac{\rho_{r}}{\Phi}\right)} \\
& {\left[{ }^{226} \mathrm{Ra}_{w}-\left({ }^{226} \mathrm{Ra}_{w(0)} \cdot \mathrm{e}^{-\lambda_{226} \cdot \mathrm{R}_{R a-226} \cdot T}\right)\right]=\left[\frac{\left(\Delta_{226}+\Gamma_{226}\right)}{\left(\lambda_{226} \cdot \mathrm{R}_{R a-226}\right)}\right] \cdot\left(1-\mathrm{e}^{-\lambda_{226} \cdot \mathrm{R}_{R a-226} \cdot T}\right) \cdot\left({ }^{226} \mathrm{Ra}_{r} \cdot \frac{\rho_{r}}{\Phi}\right)}
\end{aligned}
$$

$$
\left[\frac{{ }^{228} \mathrm{Ra}_{w}-\left({ }^{228} \mathrm{Ra}_{w(0)} \cdot \mathrm{e}^{-\lambda_{228} \cdot \mathrm{R}_{\mathrm{Ra}-228} \cdot T}\right)}{{ }^{226} \mathrm{Ra}_{w}-\left({ }^{226} \mathrm{Ra}_{w(0)} \cdot \mathrm{e}^{-\lambda_{226} \cdot \mathrm{R}_{\mathrm{Ra}-228} \cdot T}\right)}\right] \cdot \frac{{ }^{226} \mathrm{Ra}_{r}}{{ }^{228} \mathrm{Ra}_{r}}=\left[\frac{\left(\Delta_{228}+\Gamma_{228}\right)}{\left(\Delta_{226}+\Gamma_{226}\right)}\right] \cdot \frac{\lambda_{226}}{\lambda_{228}} \cdot \frac{\left(1-\mathrm{e}^{-\lambda_{228} \cdot \mathrm{R}_{\mathrm{Ra}-228} \cdot T}\right)}{\left(1-\mathrm{e}^{-\lambda_{226} \cdot \mathrm{R}_{\mathrm{Ra}-228} \cdot T}\right)}
$$




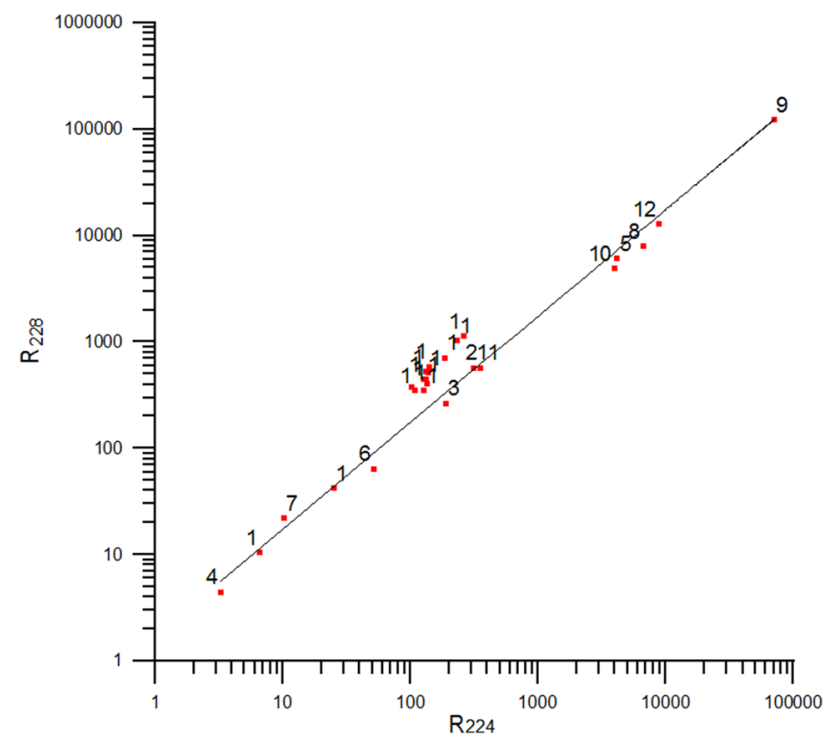

Fig. 4 Radium retardation factors from literature: $\mathrm{R}_{\mathrm{Ra}-228}$ and $\mathrm{R}_{\mathrm{Ra}-224}$ in groundwater samples from 12 different aquifers. Samples from aquifers 1-7: data in [4]; from aquifers 8-10: data in [10]; from aquifers 11-12: data in $[36,37]$
Equation 19 has the great advantage of not being dependent on $\Phi$ and $\rho_{\mathrm{r}}$ of the aquifer rock. Assuming $\Delta_{228}=\Delta_{226}$ (congruent dissolution), three typical cases for Eqs. 17-19 are developed (Eqs. 41-52, Appendix 5).

The $\mathrm{R}_{\mathrm{Ra}-228}, \mathrm{R}_{\mathrm{Ra}-226}$ and the $\Gamma_{228}, \Gamma_{226}$ found by the NAPL method will be used to estimate the residence time of groundwater hosted in Red Pozzolane (RP) aquifer of the volcanic region of Alban Hills, Rome (Fig. 5). The RP hydrogeological complex consists of massive and chaotic pyroclastic with mean thickness of $20 \mathrm{~m}$. It is characterized by a medium to medium-high permeability due to a high porosity locally diminished by zeolitization. The complex constitutes the main aquifer of Rome, particularly in the area on the left bank of Tiber river, where it is spread with continuous and relevant thicknesses [42]. Scoria clasts in the RP flow deposits are scarcely porphyritic containing millimetre-sized fresh leucite with subordinate clinopyroxene. Primary leucite is often replaced with analcime [43]. The groundmass is largely formed by glass turned to zeolites and/or halloysite and by leucite microcrysts with star-like habit [44]. The abundance of leucite required a study of Ra adsorption onto this mineral. As application of

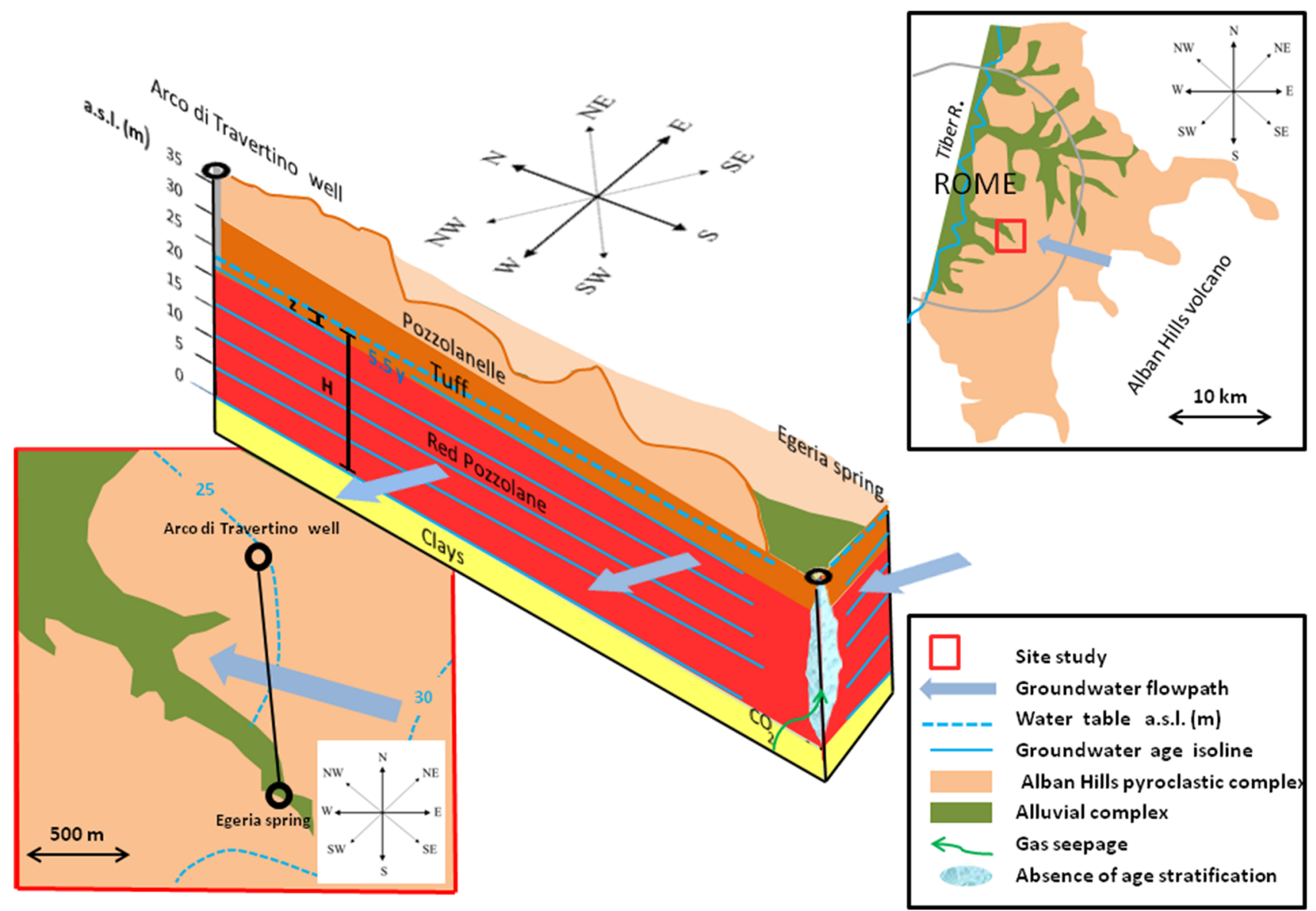

Fig. 5 Geological section and hydrogeological setting of the Red Pozzolane Aquifer (Alban Hills, Rome) 
the NAPL method, we describe an attempt of measuring the age of groundwater from a piezometer well and from a mineral spring located in proximity of the peripheral part of the PR hydrogeological complex. Initially, however, some experimental evidence of the validity of the assumptions of the NAPL method will be provided.

\section{Materials, methods and experimental procedures}

Tests on different materials, normally adsorbing Ra from aqueous solutions, were carried out to validate the assumption of negligible effects of Ra adsorption at the interface between NAPL and mineral grains. Manganese dioxide, zeolite $4 \mathrm{~A}$ and natural clays were selected for Ra adsorption experiments using aqueous and organic solutions enriched in radium isotopes. Monazitic sand and a natural Th-enriched weathered volcanic rock were selected for testing the reliability of assuming retardation factors equal to unity for Ra and even for Th ions when aquifer rock is immersed in NAPLS for a long time. Finally Ra adsorption experiments on leucite, carried out in IGAGlab in the past and never published, are presented as an aid for discussing experimental data obtained for groundwater of RP aquifer.

The size distribution of samples was obtained in wet mode by a laser particle size analyser, model Sympatec Helos, equipped with He-Ne laser source (Supplementary material).

XRPD analysis was carried out with a fully automated Bruker AXS D8 advance diffractometer, operating in reflection mode with $\theta-\theta$ geometry, equipped with high-resolution energy-dispersive 1-D Linxeye XE detector. Measurement parameters used were: $\mathrm{Cu} \mathrm{Ka}, 40$ kV, 30 mA, scan angle $(2 \theta)=5^{\circ}-80^{\circ}$; step width $(2 \theta)=0.020^{\circ}$.

Determination of $\mathrm{Ra}$ isotopes in solids and liquids was carried out by high-resolution $\gamma$-spectrometry with an EG\&G Ortec solid-state photon detector (HPGe) and Coaxial detector system, following procedures and standardization methods described in a previous paper [2]. Samples were sealed in Rn-tight containers (to avoid Rn escaping). Ra isotopes were measured from the $\gamma$-emission of their daughters after waiting the conditions of radioactive equilibrium. In particular ${ }^{226} \mathrm{Ra},{ }^{228} \mathrm{Ra}$ and ${ }^{224} \mathrm{Ra}$ were determined from $\gamma$-emission of ${ }^{214} \mathrm{~Pb},{ }^{228} \mathrm{Ac}$ and ${ }^{212} \mathrm{~Pb}$, respectively. Radioactive equilibrium between ${ }^{226} \mathrm{Ra}$ and ${ }^{214} \mathrm{~Pb}$ was reached in 20 days, while between ${ }^{228} \mathrm{Ra}$ and ${ }^{228} \mathrm{Ac}$ in 1 day and between ${ }^{224} \mathrm{Ra}$ and ${ }^{212} \mathrm{~Pb}$ in 2 days.

${ }^{224} \mathrm{Ra}$ was determined after correction for decay occurring between separation time and initial counting and during $\gamma$-counting. In some experiments, ${ }^{212} \mathrm{~Pb}$ was also measured directly for determining the adsorption of lead.
${ }^{220} \mathrm{Rn}$ was measured following the method named "Rad $\mathrm{H}_{2} \mathrm{O}^{\prime \prime}$ using a continuous Rn Gas Monitor model Rad 7, Durridge Company, equipped with a solid-state ion-implanted planar silicon detector [45].

Kerosene (Alfa Aesar) and pure n-hexane (Merck) were chosen as apolar fluids representative of light NAPLs. They display similar apolarity but only kerosene, due to its complex nature, contains also organic polar compounds (e.g. naphtenic acids). They differ notably by volatility and by cost as well. Both were previously checked by $\gamma$-spectrometry, for excluding the detectable presence of Ra isotopes.

Th-Welsbach mantles [46] were immersed for 20 days in $50 \mathrm{ml}$ of kerosene obtaining a ${ }^{224} \mathrm{Ra}$-enriched kerosene phase (K20D) and for 2 years in $50 \mathrm{ml}$ of kerosene obtaining a ${ }^{228} \mathrm{Ra}-{ }^{224} \mathrm{Ra}$-enriched kerosene phase (K2Y). From aqueous Ra solution, obtained by chromatographic separation of Ra from standard Th solution (Spectrascan Analytical Standards), ${ }^{228}$ Ra was extracted by synergistic complexation with TTA (Thenoyltrifluoroacetone) and TOPO (Trioctylphosphine oxide) in $\mathrm{n}$-hexane [47] producing an organic phase $(\mathrm{RaC})$ containing complexed radium. K20D, $\mathrm{K} 2 \mathrm{Y}$ and $\mathrm{RC}$ were used in experiments after measurement of their Ra activity by $Y$-spectrometry. Table 1 resumes the plan of the experiments carried out in this work.

\section{1 $\mathrm{Ra}$ adsorption on $\mathrm{MnO}_{2}$ in kerosene and recoil constant of ${ }^{224} \mathrm{Ra}$ recoiled from ${ }^{228} \mathrm{Th}$ adsorbed on $\mathrm{MnO}_{2}$}

$\mathrm{MnO}_{2}$ was selected for its known capacity of adsorbing bivalent cations in aqueous solutions [48]. Thus, it can be useful to compare Ra adsorption in water and in NAPL. The adsorption kinetics in water is fast: after $30 \mathrm{~min}$ about $90 \%$ of $\mathrm{Pb}$ and $50 \%$ of $\mathrm{Ra}$ are removed by adsorption on $\mathrm{MnO}_{2}$ [48-50]. The $<0.125 \mathrm{~mm}$ fraction of manganese dioxide black powder (Carlo Erba, BET surface area: $4 \mathrm{~m}^{2} \mathrm{~g}^{-1}$ ), MDBP, was selected for Ra adsorption experiments. Particle bulk density $\rho_{r}$ and porosity $\Phi$ of MDBP were evaluated according standard procedures [51].

\subsubsection{Experiments MDBP-1 $\mathrm{K}$ and MDBP-1H}

This experiment was carried out to test the adsorption of bivalent simple ions like $\mathrm{Ra}^{2+}$ and $\mathrm{Pb}^{2+}$ when $\mathrm{MnO}_{2}$ is immersed in NAPL (kerosene and n-hexane). $10 \mathrm{ml}$ of K2Y were added to $0.1 \mathrm{~g}$ of MDBP (particle /liquid ratio $\left.=0.01 \mathrm{~kg} \cdot \mathrm{L}^{-1}\right)$. After agitation for $30 \mathrm{~min}$, the organic phase (MDBP-1 K) was separated by centrifugation and suddenly counted for ${ }^{212} \mathrm{~Pb}$ while ${ }^{228}$ Ra was detected after 1 day. $10 \mathrm{ml}$ of $\mathrm{n}$-hexane were added to $0.1 \mathrm{ml}$ of liquid resulting from evaporation of $10 \mathrm{ml}$ of $\mathrm{K} 2 \mathrm{Y}$ and counted for ${ }^{228} \mathrm{Ra}$ and ${ }^{212} \mathrm{~Pb}$. Then this $\left({ }^{228} \mathrm{Ra},{ }^{212} \mathrm{~Pb}\right)$-enriched 
Table 1 Experiment plan

\begin{tabular}{|c|c|c|c|c|c|}
\hline SAMPLE & EXPERIMENT & CONTACT TIME & $\begin{array}{l}\text { ANALYSED } \\
\text { PHASE }\end{array}$ & DETECTED ISOTOPES & $\begin{array}{l}\text { MAIN RESULTS AND } \\
\text { DERIVED PARAMETERS }\end{array}$ \\
\hline MDBP-1 K & $\mathrm{MDBP}+\mathrm{K} 2 \mathrm{Y}$ & $30 \mathrm{~min}$ & Ker & ${ }^{212} \mathrm{~Pb},{ }^{228} \mathrm{Ra}$ & moder. Pb ads./ moder. Ra ads \\
\hline MDBP-1H & $\mathrm{MDBP}+\mathrm{K} 2 \mathrm{Y}+\mathrm{Hex}$ & $30 \mathrm{~min}$ & Hex $+($ Ker $)$ & ${ }^{212} \mathrm{~Pb},{ }^{228} \mathrm{Ra}$ & neglig. $\mathrm{Pb}$ ads./ neglig. $\mathrm{Ra}$ ads \\
\hline MDBP-2 & $\mathrm{MDBP}+\mathrm{RaC}+\mathrm{Ker}$ & $30 \mathrm{~min}$ & Ker + Hex & ${ }^{228}$ Ra-complex & high Ra adsorption \\
\hline MDBP-3a & Th-MDBP + Ker & 20 days & Ker & ${ }^{224} \mathrm{Ra}$ & $f_{a a 224}-\Gamma_{\mathrm{Ra}}(\mathrm{NAPL}$ method) \\
\hline MDBP-3b & Th-MDBP + Water & $5 \min$ & Water & ${ }^{220} \mathrm{Rn}$ & $f_{a 220}-\Gamma_{\mathrm{Rn}-220}$ \\
\hline Z4A-1 & $\mathrm{Z} 4 \mathrm{~A}+\mathrm{K} 20 \mathrm{D}$ & 2 days & Ker & ${ }^{224} \mathrm{Ra}$ & negligible Ra adsorption \\
\hline Z4A-2 & $\mathrm{Z} 4 \mathrm{~A}+\mathrm{RaC}+\mathrm{Ker}$ & 2 days & Ker + Hex & ${ }^{228}$ Ra-complex & moderate Ra adsorption \\
\hline $\mathrm{Z} 4 \mathrm{~A}-3$ & $\mathrm{Z} 4 \mathrm{~A}+{ }^{228} \mathrm{Ra}-$ Water & 2 days & Water & ${ }^{228} \mathrm{Ra}$ & high Ra adsorption \\
\hline S8N1-1 K & S8N1 + K20D & 1 days & Ker & ${ }^{224} \mathrm{Ra}$ & negligible Ra adsorption \\
\hline S8N1-3 K & S8N1 + K20D & 3 days & Ker & ${ }^{224} \mathrm{Ra}$ & negligible Ra adsorption \\
\hline S8N1-3 W & $\mathrm{S} 8 \mathrm{~N} 1+{ }^{228} \mathrm{Ra}-$ Water & 3 days & Water & ${ }^{228} \mathrm{Ra}$ & high Ra adsorption \\
\hline MON-1 K & monazite + Ker & 2 days & Ker & ${ }^{224} \mathrm{Ra}$ & $f_{a 224}-\Gamma_{\mathrm{Ra}}$ (NAPL method) \\
\hline MON-1 K & monazite + Ker & 27 months & Ker & ${ }^{228} \mathrm{Th},{ }^{228} \mathrm{Ra}$ & Treal三Tmeasured(i.e.noadsorption) \\
\hline CMB-1 K & $\mathrm{CM}+\mathrm{Ker}$ & 2 years & Ker & ${ }^{228} \mathrm{Ra}$ & $\mathrm{R}_{\mathrm{Ra}-228}$ (NAPL method) \\
\hline CMB-1 W & $\mathrm{CM}+$ Water & 2 years & Water & ${ }^{228} \mathrm{Ra}$ & $\begin{array}{l}\text { Treal } \equiv \text { Tmeasured (i.e. no adsorption } \\
\mathrm{R}_{\mathrm{Ra}-228} \text { (NAPL method) }\end{array}$ \\
\hline CM-1aK & $\mathrm{CM}-1+\mathrm{Ker}$ & 20 days & Ker & ${ }^{224} \mathrm{Ra}$ & $\mathrm{R}_{\mathrm{Ra}-224}$ (NAPL method) \\
\hline CM-1bW & CM-1 + Water & 20 days & Water & ${ }^{224} \mathrm{Ra}$ & $\mathrm{R}_{\mathrm{Ra}-224}$ (NAPL method) \\
\hline CM-1cW & CM-1 + Water & $5 \mathrm{~min}$ & Water & ${ }^{220} \mathrm{Rn}$ & $f_{220}-\Gamma_{\mathrm{Rn}-220}$ \\
\hline $\mathrm{RP}-2 \mathrm{~K}$ & $\mathrm{RP}-2+\mathrm{Ker}$ & 20 days & Ker & ${ }^{224} \mathrm{Ra}$ & $\mathrm{R}_{\mathrm{Ra}-224}$ (NAPL method) \\
\hline $\mathrm{RP}-2 \mathrm{~W}$ & RP-2 + Water & 20 days & Water & ${ }^{224} \mathrm{Ra}$ & $\mathrm{R}_{\mathrm{Ra}-224}$ (NAPL method) \\
\hline RP-2R & RP-2 + Water & $5 \min$ & Water & ${ }^{220} \mathrm{Rn}$ & $f_{220}-\Gamma_{\mathrm{Rn}-220}$ \\
\hline
\end{tabular}

Ker $=$ Kerosene; moder $=$ moderate; neglig. $=$ negligible

hexane was added to $0.1 \mathrm{~g}$ of MDBP (particle /liquid ratio $\left.=0.0099 \mathrm{~kg} \cdot \mathrm{L}^{-1}\right)$ and stirred for $30 \mathrm{~min}$. Finally the organic phase (MDBP-1H) was separated and counted for ${ }^{212} \mathrm{~Pb} .{ }^{228}$ Ra was.

detected after 1 day.

\subsubsection{Experiment MDBP-2}

The experiment is aimed to highlight the eventual different adsorption on $\mathrm{MnO}_{2}$ when Ra occurs as organic complex (RaC:TOPO-TTA Ra complex). RaC organic phase was mixed with kerosene (1:2 volume ratio) and counted after 1 day for detecting initial ${ }^{228} \mathrm{Ra}$. Then $15 \mathrm{ml}$ of this mixture were added to $0.1 \mathrm{~g}$ of MDBP (particle /liquid ratio $=0.0067 \cdot 10^{-2} \mathrm{~kg} \cdot \mathrm{L}^{-1}$ ) and, after $30 \mathrm{~min}$ of agitation, the organic phase (MDBP-2) was separated and counted for ${ }^{228}$ Ra after 1 day.

\subsubsection{Experiment MDPB-3}

The following experiment was used to test whether the radon emanation of ${ }^{220} \mathrm{Rn}, f_{220}$, approximates the alpha recoil fraction of ${ }^{224} \mathrm{Ra}, f_{\alpha-224}$, measured by NAPL method, when parent radionuclides $\left({ }^{224} \mathrm{Ra}\right.$ and ${ }^{228} \mathrm{Th}$, respectively) are distributed in the same way exclusively as adsorbed atoms on particle grains. $10 \mathrm{ml}$ of a standard Th solution in nitric acid (1000 ppm of ${ }^{232} \mathrm{Th}$ ) were slowly dried on $16.0 \mathrm{~g}$ of MDBP by continuous stirring at $T<80^{\circ} \mathrm{C}$. The $\mathrm{HNO}_{3}$ treatment greatly increases the surface area [52]. In these conditions, Th nitrate (density $=2.8 \mathrm{~g} \cdot \mathrm{cm}^{-3}$ ) should cover the $\mathrm{MnO}_{2}$ grains (specific surface $=4 \cdot 10^{4} \mathrm{~cm}^{2} \mathrm{~g}^{-1}$ ) with a thickness $<0.2 \mathrm{~nm}$. This is a trascurable fraction of the $R_{c}$ in Th nitrate $\left(R_{c}=30 \mathrm{~nm}\right)$. In this way ${ }^{228}$ Th occurs only as adsorbed ion, allowing ${ }^{224}$ Ra-recoil exclusively from the surface of grains. At dryness, $8 \mathrm{~g}$ of Th-enriched MDBP were immersed in $30 \mathrm{ml}$ of kerosene ( particle /liquid ratio $=0.27 \mathrm{~kg} \cdot \mathrm{L}^{-1}$ ) up to 20 days to reach radioactive equilibrium between surfacial ${ }^{228} \mathrm{Th}$ and recoiled ${ }^{224} \mathrm{Ra}$. Then kerosene (MDBP-3a) was separated by centrifugation and filtration and counted after 2 days for ${ }^{224} \mathrm{Ra}$. Another subsample of Th-enriched MDBP ( $8 \mathrm{~g})$ was immersed in $30 \mathrm{ml}$ of water (particle /liquid ratio $=0.27 \mathrm{~kg} \cdot \mathrm{L}^{-1}$ ) and ${ }^{220} \mathrm{Rn}$ activity in water (MDBP-3b) was measured.

\subsection{Ra adsorption on zeolite 4A}

The importance of these experiments relies on the evidence that in many volcanic aquifers, like RP aquifer, 
zeolites are important Ra-sinking mineral phases. We have used zeolites of A family, known as LTA (Linde Type A), which are extensively used as standard Ra adsorbers $[53,54]$. Zeolite $4 \mathrm{~A}$ (Bis Italia s.r.l.) was used in these experiments. In zeolite $4 A$, negative charges, formed in the tetrahedral framework, are compensated by exchangeable $\mathrm{Na}^{+}$.

The first experiment (Z4A-1) tests Ra adsorption (as simple ion) on zeolite immersed in NAPL. The second experiments (Z4A-2) tests adsorption on zeolite in NAPL when Ra occurs as organic complex (RaC:TOPO-TTA Ra complex). A third experiment (Z4A-3) was carried out to compare Ra adsorption (as simple ion) on zeolite when immersed in water.

Ten $\mathrm{ml}$ of K20D were added to $2 \mathrm{~g}$ of zeolite $4 \mathrm{~A}$ (particle /liquid ratio $=0.2 \mathrm{~kg} \cdot \mathrm{L}^{-1}$ ) and stirred for 2 days. Then kerosene (Z4A-1) was separated and after 2 days was counted for ${ }^{224} \mathrm{Ra}$.

$5 \mathrm{ml}$ of RaC were mixed with $20 \mathrm{ml}$ of kerosene and counted after 1 day for detecting ${ }^{228} \mathrm{Ra}$. Then the resulting organic phase was added to $2 \mathrm{~g}$ of zeolite $4 \mathrm{~A}$ (particle /liquid ratio $=0.08 \mathrm{~kg} \cdot \mathrm{L}^{-1}$ ). After 2 days of stirring, the organic phase (Z4A-2) was separated and, after 1 day, counted for ${ }^{228} \mathrm{Ra}$.

$20 \mathrm{ml}$ of water solution ( $\mathrm{pH} 5.5$ ), containing a known amount of ${ }^{228} \mathrm{Ra}$, obtained by chemical separation from Th standard solution, was added to $2 \mathrm{~g}$ of zeolite $4 \mathrm{~A}$ (particle /liquid ratio $=0.1 \mathrm{~kg} \cdot \mathrm{L}^{-1}$ ) and stirred for 2 days. Then water (Z4A-3) was separated and, after 1 day, measured for ${ }^{228}$ Ra.

\subsection{Ra adsorption on clay}

The pliocenic clay of Dunarobba fossil forest $[55,56]$, containing numerous clay minerals as montmorillonite, saponite, mixed layered chlorite-montmorillonite, illite, chlorite and kaolinite, was chosen for adsorption experi- liquid ratio $\left.=0.03 \mathrm{~kg} \cdot \mathrm{L}^{-1}\right) .(\mathrm{ml} / \mathrm{g}=33.3)$, were stirred for 1 and 3 days, respectively. Then kerosene phases were separated by centrifugation and counted for ${ }^{224} \mathrm{Ra}$ after 2 days.

$25 \mathrm{ml}$ of an aqueous solution ( $\mathrm{pH} 7.0$ ) containing a known amount of ${ }^{228} \mathrm{Ra}\left(3.8 \cdot 10^{3} \mathrm{~Bq} \cdot \mathrm{L}^{-1}\right)$, obtained by chemical separation from Th standard solution, was added to $0.75 \mathrm{~g}$ of $S 8 \mathrm{~N} 1$ (particle /liquid ratio $=0.03 \mathrm{~kg} \cdot \mathrm{L}^{-1}$ ) and stirred for 3 days. Then water (S8N1-3 W) was separated by centrifugation and, after 1 day, ${ }^{228}$ Ra activity was measured.

\subsection{Monazite in contact with NAPL}

This experiment was carried out to test the validity of assuming $R_{R a}$ and $R_{T h}$ in NAPL (kerosene) equal to 1 , by measuring the ${ }^{228} \mathrm{Th} /{ }^{228} \mathrm{Ra}$ age of contact between monazite and NAPL.

$10 \mathrm{ml}$ of kerosene was added to $35 \mathrm{~g}$ of pure monazitic sand (particle /liquid ratio $=0.29 \mathrm{~kg} \mathrm{~L}^{-1}$ ). After 20 days of stirring, kerosene $\left(\mathrm{MON}^{-1} \mathrm{~K}\right)$ was separated and after 2 days ${ }^{224}$ Ra activity was measured for determining $f_{a 224}$. After the $\gamma$-counting, kerosene was newly put in contact with the monazitic sand up to 27 months. Then kerosene was separated and, after 2 weeks, ${ }^{228}$ Ra and ${ }^{228} \mathrm{Th}$ (the latter one through ${ }^{224} \mathrm{Ra}$ in secular equilibrium with ${ }^{228} \mathrm{Th}$ ) were measured. Monazite does not dissolve in kerosene, thus the ${ }^{228} \mathrm{Th} /{ }^{228}$ Ra ratio in kerosene depends only on a-recoil phenomena. The temporal variation of this radioactive pair is described by the following equation when retardation factor of Th and $\mathrm{Ra}$ are both equal to 1 [58]:

$\frac{{ }^{228} \mathrm{Th}}{{ }^{228} \mathrm{Ra}}=\frac{1-\mathrm{e}^{-\lambda_{\mathrm{Th}-228} \cdot T}-\left[\left(\frac{\lambda_{\text {Th }-228}}{\lambda_{\mathrm{Th}-228}-\lambda_{\text {Ra }-228}}\right) \cdot\left(\mathrm{e}^{-\lambda_{\text {Ra }-228} \cdot T}-\mathrm{e}^{-\lambda_{\text {Th }-228} \cdot T}\right)\right]}{1-\mathrm{e}^{-\lambda_{\text {Ra }-228} \cdot T}}$

Instead, considering explicitly retardation factors of Th and Ra, Eq. 20 becomes:

$\frac{{ }^{228} \mathrm{Th}}{{ }^{228} \mathrm{Ra}}=\frac{\mathrm{R}_{\mathrm{Ra}}}{\mathrm{R}_{\mathrm{Th}}} \cdot \frac{1-\mathrm{e}^{-\lambda_{\mathrm{Th}-228} \cdot T \cdot \mathrm{R}_{\mathrm{Th}}}-\left[\left(\frac{\lambda_{\mathrm{Th}-228} \cdot \mathrm{R}_{\mathrm{Th}}}{\lambda_{\mathrm{Th}-228} \cdot \mathrm{R}_{\mathrm{Th}}-\lambda_{\text {Ra }-228} \cdot \mathrm{R}_{\text {Ra }}}\right) \cdot\left(\mathrm{e}^{-\lambda_{\text {Ra }}-228 \cdot T \cdot \mathrm{R}_{\text {Ra }}}-\mathrm{e}^{-\lambda_{\mathrm{Th}}-228 \cdot T \cdot \mathrm{R}_{\mathrm{Th}}}\right)\right]}{1-\mathrm{e}^{-\lambda_{\text {Ra }}-228} \cdot T \cdot \mathrm{R}_{\text {Ra }}}$

ments on clay. The sample S8N1 (60-120 mesh fraction), with a low content of organic matter (3\%), was selected for its negligible ${ }^{224} \mathrm{Ra}$ activity $\left(0.01 \mathrm{~Bq} \mathrm{~g}^{-1}\right)$ in secular equilibrium with ${ }^{228}$ Th [57].

Samples S8N1-1 K and S8N1-3 K, prepared in the same way, by adding $25 \mathrm{ml}$ of $\mathrm{K} 20 \mathrm{D}$ to $0.75 \mathrm{~g}$ of S8N1 (particle /
At secular equilibrium, Eq. 21 predicts that ${ }^{228} \mathrm{Th} /{ }^{228} \mathrm{Ra}=\mathrm{R}_{\mathrm{Ra}} / \mathrm{R}_{\mathrm{Th}}[59]$.

It's clear that only if both $R_{R a}$ and $R_{T h}$ in kerosene approximate to 1 , the age of MON-1 K, calculated by Eq. 20, corresponds to the true one (27 months). 


\subsection{Application of the NAPL method for determining Ra retardation factor and recoil constant for a Th-enriched weathered volcanic rock}

A Th-enriched, highly porous pyroclastic rock, composed by diatomite beds mixed with deeply altered leucititictephrite in the quarry of Casale Morticini (CM) near Montefiascone (Vulsini volcano, Latium, Italy) was selected. In this area, subduction-related metasomatic enrichment of incompatible elements in the mantle source, coupled with magma differentiation within the upper crust, gave rise to $\mathrm{U}, \mathrm{Th}$ and K-enriched melts [60]. Alteration by $\mathrm{CO}_{2}$ and $\mathrm{H}_{2} \mathrm{~S}$-rich gaseous exhalation resulted in abundant amorphous silica with scarce halloysite $10 \AA$ and allophane [61]. The XRD spectrum of the sample (see Supplementary material) reveals hydrothermal quartz and jarosite among main minerals, as in similar degassing areas of Latium [62]. A broad halo between $20^{\circ}-30^{\circ}(2 \theta)$ indicates that the bulk of material is amorphous. The ${ }^{232} \mathrm{Th}$ activity of $\mathrm{CM}$, measured by high resolution $\gamma$-spectrometry, is close to $1,980 \mathrm{~Bq} \mathrm{~kg}^{-1}$ (500 ppm Th), about five times the average of Vulsini volcanic products [60].

\subsubsection{Experiments CMB-1 K and CMB-1 W}

This experiment tests the capacity of NAPL method to find directly $R_{\text {Ra-228 }}$ and the validity of assuming $R_{R a}$ and $R_{T h}$ in kerosene equal to 1 by measuring the resulting ${ }^{228} \mathrm{Th} /{ }^{228} \mathrm{Ra}$ age of contact between volcanic rock and NAPL. CM was dryed at $105^{\circ} \mathrm{C}$ and splitted in two portions of $50 \mathrm{~g}$. They were saturated and rinsed with $225 \mathrm{ml}$ of kerosene (CMB-1 K) and distilled water (CMB-1 W), respectively, using flasks of $250 \mathrm{cc}$ (particle /liquid ratio $=0.222 \mathrm{~kg}$ $\left.\cdot \mathrm{L}^{-1}\right)$. After 2 years, the liquid phases were separated and their ${ }^{228} \mathrm{Ra}$ activities determined. The ${ }^{228} \mathrm{Th}$ activity of sample CMB-1 $\mathrm{K}$ was also measured.

\subsubsection{Experiments $\mathrm{CM}-1 \mathrm{aK}$, $\mathrm{CM}-1 \mathrm{bW}, \mathrm{CM}-1 \mathrm{cW}$}

These experiments have the objective to compare the NAPL method with the Rn method in determining Ra retardation factor. NAPL method on ${ }^{224}$ Ra was carried out on subsample (CM-1), obtained by sieving a pulverized sample of $C M$ and selecting the $<125 \mu$ fraction (120 ASTM mesh). This for working with a sample having a more defined granulometry and also higher in thorium, which generally enriches in fine fraction. The fraction displayed a measured ${ }^{232} \mathrm{Th}$ activity of $2,900 \mathrm{~Bq} \mathrm{~kg}^{-1}$ (732 ppm). Particle bulk density, porosity and distribution size of CM-1 (see Supplementary material) were determined and $18 \mathrm{~g}$ of CM-1 were immersed in $41 \mathrm{ml}$ of kerosene (particle /liquid ratio $=0.44 \mathrm{~kg} \cdot \mathrm{L}^{-1}$ ) for 20 days. Successively kerosene was separated (CM-1aK) and after 2 days the ${ }^{224} \mathrm{Ra}_{\text {NAPL }}$ activity was measured. A portion of CM-1 (18 g) was immersed in $41 \mathrm{ml}$ of distilled water (particle /liquid ratio $=0.44 \mathrm{~kg}$ $\left.\cdot \mathrm{L}^{-1}\right)$ for 20 days. Then water was separated (CM-1bW) and detected for ${ }^{224} \mathrm{Ra}_{\mathrm{w}}$ after 2 days. Finally, $18 \mathrm{~g}$ of CM-1 were immersed in $250 \mathrm{ml}$ of distilled water (CM-1 cW, particle / liquid ratio $=0.072 \mathrm{~kg} \cdot \mathrm{L}^{-1}$ ) and the ${ }^{220} \mathrm{Rn}$ emanation coefficient $\left(f_{220}\right)$ in water was measured.

\subsection{Ra adsorption onto leucite}

${ }^{226} \mathrm{Ra}$ adsorption on leucite at ambient temperature was studied. A solution of known ${ }^{226}$ Ra activity, deriving from extraction and chemical separation of uraninite (Black Hills), was used for adsorption experiments. Five samples, each one composed by several leucite crystals from Pozzolanelle Unit of Alban Hills volcanic district [63] were selected on the basis of their size. The composite total surface area of each sample was determined. They were immersed in different volumes of an aqueous ${ }^{226} \mathrm{Ra}$-solution of known activity at $\mathrm{pH} 7.0\left(\mathrm{~T}=298^{\circ} \mathrm{K}\right)$ and stirred for 1 week. Then aqueous solutions were separated and kept in sealed containers for 20 days before of determining the desorbed ${ }^{226}$ Ra activity.

\subsection{Study site: $\mathrm{Ra}$ isotopes in groundwater from the Red Pozzolane aquifer (Alban Hills)}

$40 \mathrm{~L}$ of groundwater (RP-1) of the aquifer hosted in the RP hydrological unity were extracted from a piezometric well (Fig. 5) located near the Arco di Travertino (Appia road, Rome). Groundwater was sampled at a depth of $12 \mathrm{~m}$ from ground level and about $1.5 \mathrm{~m}$ below the local water table.

$20 \mathrm{~g}$ of $\mathrm{Ba}(\mathrm{NO} 3)_{2}$ and a calculated excess of $\mathrm{H}_{2} \mathrm{SO}_{4}$ were added to RP-1 for precipitating ( $\mathrm{Ba}, \mathrm{Ra}$ ) sulphate. The efficiency of precipitation was obtained by the weight ratio of recovered $\mathrm{BaSO}_{4}$ to the added amount of $\mathrm{Ba}(\mathrm{NO} 3)_{2}$ [64]. Dry powder from precipitation was measured after 2 days for detecting ${ }^{224} \mathrm{Ra}$ and ${ }^{228} \mathrm{Ra}$. The powder was then sealed in an air-tight container and, after 20 days, ${ }^{226} \mathrm{Ra}$ was measured.

$192 \mathrm{~g}$ of RP (RP-2) were measured for Ra isotopes by $\mathrm{Y}$-spectrometry. Then two portions of RP- 2 were treated by NAPL method using kerosene (RP-2 K) and water (RP-2 W) $(\mathrm{ml} / \mathrm{g}=0.36)$. A third aliquot of RP-2 was immersed in a 250-ml flask filled with distilled water (RP-2R) and the ${ }^{220} \mathrm{Rn}$ emanation in water $\left(f_{220}\right)$ was measured. Since the high hydrolytic reactivity of RP formation is well known [65], its dissolution at the first stage of contact with water was studied experimentally. RP was reacted with Millipore water $(\mathrm{ml} / \mathrm{g}=2.5)$ at $1 \mathrm{~atm}$ in a constant-temperature water bath at $298^{\circ} \mathrm{K}$ for a total time of 45 days. The mixture was agitated magnetically at intervals of $1 \mathrm{~h}$ (during the first 


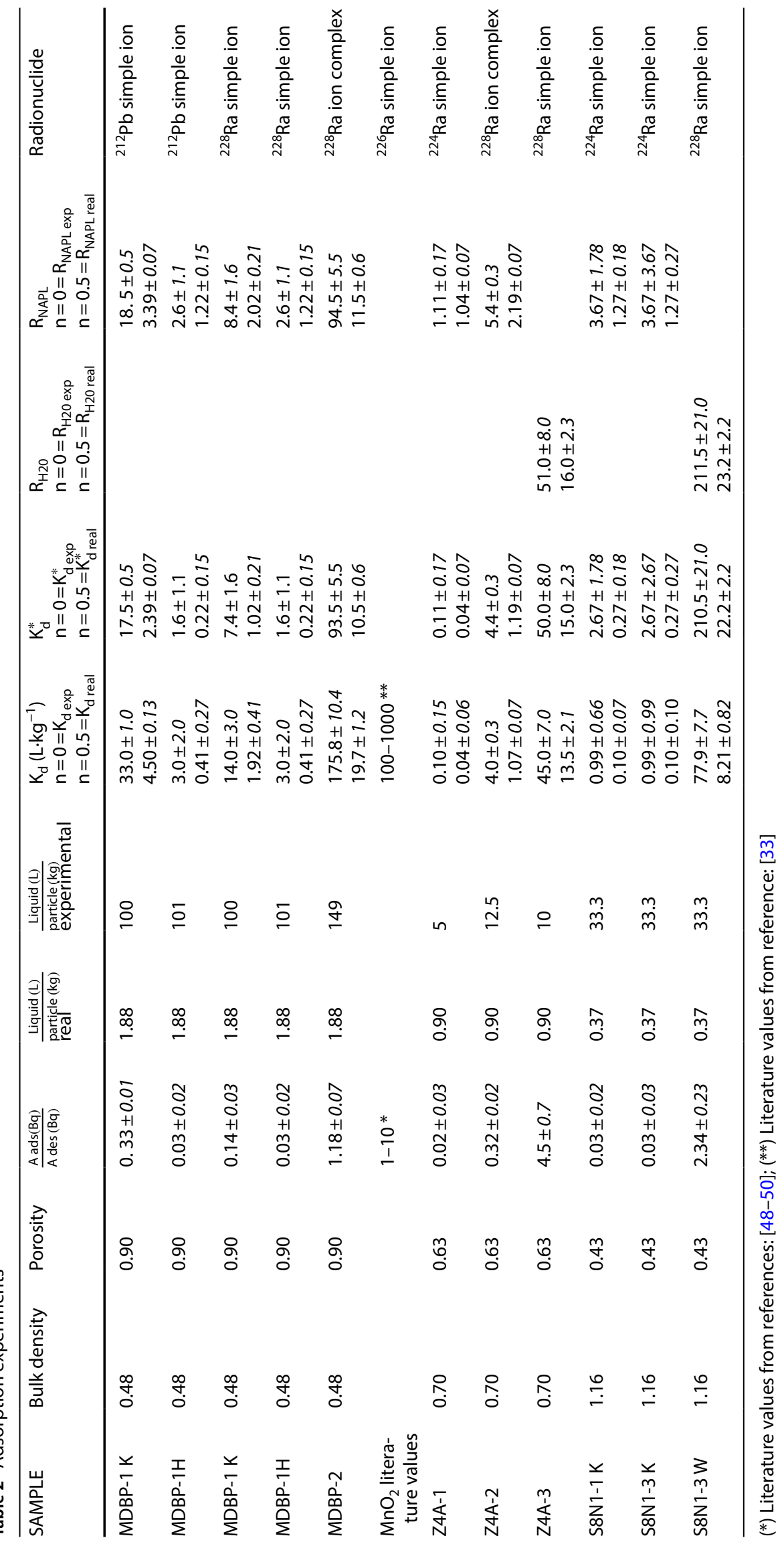


day) and successively of $24 \mathrm{~h}$. The extent of initial dissolution of the rock was monitored by a $\mathrm{pH}$ meter $(\mathrm{pH} 340-$ A, WTW, Germany) and an electrical conductivity meter (LF340, WTW, Germany). The probes were kept in continuous contact with solution and values recorded periodically. Electrical conductivity was converted, after calibration, in terms of TDS of solution and expressed as TDSL of pore water according the formula:

TDSL $=$ TDS of solution $\cdot(250 / 100) \cdot\left(\rho_{d} / \Phi\right)$.

\section{Results and discussion}

Results of adsorption experiments are reported in Table 2. The latter ones are expressed in terms of the dimensional and dimensionless adsorption distribution coefficients, $\mathrm{K}_{\mathrm{d} \text { exp }}\left(\mathrm{L} \cdot \mathrm{kg}^{-1}\right)$ and $\mathrm{K}_{\mathrm{d} \text { exp }}^{*}$ respectively. $\mathrm{K}_{d \exp }$ was calculated by the following equation:

$\mathrm{K}_{\text {dexp }}=\frac{\text { Activity adsorbed }(\mathrm{Bq})}{\text { Activity desorbed }(\mathrm{Bq})} \cdot\left(\frac{\text { Liquid }(\mathrm{L})}{\text { particle }(\mathrm{kg})}\right)_{\exp }$

where $\left(\frac{\text { Liquid }(L)}{\text { particle }(\mathrm{kg})}\right)_{\exp }$ is the ratio between liquid (water or NAPL) to solid during the experiments given in Sect. 4.1, whereas $\mathrm{K}_{\text {dexp }}$ is calculated by Eq. 6. The $\left(\frac{\text { Liquid (L) }}{\text { particle }(\mathrm{kg})}\right)_{\exp }$ ratio is widely recognized as a critical component in determining retardation factors of Ra as evidenced by many authors $[33,66,67]$ that have discussed the sediment-water ratio artifact or particle concentration effect. The particle concentration effect predicts that experimental $\mathrm{K}_{d}$ differs from real $\mathrm{K}_{d}$ when $\left(\frac{\mathrm{Liquid}(\mathrm{L})}{\text { particle }(\mathrm{kg})}\right)$ exp ratio differs from real $\left(\frac{\text { Liquid }(\mathrm{L})}{\text { particle }(\mathrm{kg})}\right)$ real ratio (equal to the real $\frac{\Phi}{\rho_{r}}$ ratio) following a kind of scaling law $[68,69]$ that can be expressed as:

$\mathrm{K}_{d \text { real }}=\left[\frac{\left(\frac{\text { Liquid }}{\text { Particle }}\right)_{\text {real }}}{\left(\frac{\text { Liquid }}{\text { Particle }}\right)_{\exp }}\right]^{n} \cdot \mathrm{K}_{d \exp }$

where $n \geq 0$ is an empirical parameter called the particle effect index which measures the degree of the particle concentration effect. In Table 3, $\mathrm{K}_{\mathrm{d} \text { real }}$ and $\mathrm{K}_{\mathrm{d} \text { real }}^{*}$ values and real retardation factors calculated applying Eq. 5 are given for $n=0.5$. The value of $n=0.5$ fits very well the Ra adsorption experiments on sands provided by Beck and Cochran [33]. This value is close to the particle effect index found for $\mathrm{Zn}^{2+}$ on manganite (0.41) [69]. Such a value of particle effect index (0.5) corresponds to a dependence of adsorption on the square root of the liquid to particle ratio, and it is assumed in this work as the most reliable value for correcting distribution coefficients (expressed as $\mathrm{K}_{\mathrm{d} \text { real }}^{*}$ ) and retardation factors (expressed as $\mathrm{R}_{\text {real }}$ ) for the particle concentration effect. Results of radium analitical determinations in groundwater and rock samples together with the values of $f_{a 224}$ and $\mathrm{R}_{\mathrm{Ra}}$ obtained by applying the NAPL method are reported in Table 3.

\subsection{Ra adsorption on $\mathrm{MnO}_{2}$ in kerosene and recoil constant of ${ }^{224} \mathrm{Ra}$ recoiled from ${ }^{228} \mathrm{Th}$ adsorbed on $\mathrm{MnO}_{2}$}

\subsubsection{Experiments MDBP- $1 \mathrm{~K}$ and MDBP-1H}

In MDBP-1K and MDBP1- $\mathrm{H}$, the ratio of adsorbed ${ }^{212} \mathrm{~Pb}$ to desorbed ${ }^{212} \mathrm{~Pb}$ in kerosene and $\mathrm{n}$-hexane was equal to $0.33 \pm 0.01$ and $0.03 \pm 0.02$, respectively. $K_{\text {dreal }}^{*}$ and $\mathrm{R}_{\text {NAPL real }}$ of ${ }^{212} \mathrm{~Pb}$ vary between $2.39 \pm 0.07$ (kerosene) to $0.22 \pm 0.15$ (n-hexane) and between $3.39 \pm 007$ (kerosene) to $1.22 \pm 0.15$ ( $n$-hexane), respectively. The ratio of adsorbed ${ }^{228} \mathrm{Ra}$ to desorbed ${ }^{228} \mathrm{Ra}$ in NAPLs was equal to $0.14 \pm 0.03$ in MDBP-1K and $0.03 \pm 0.02$ in MDBP-1H. The values of $K_{\text {dreal }}^{*}$ vary between $1.02 \pm 0.21$ (kerosene) to $0.22 \pm 0.15$ ( $n$-hexane) whereas $R_{\text {NAPL real }}$ varies between $2.02 \pm 0.21$ (kerosene) to $1.22 \pm 0.15$ ( $n$-hexane), respectively. All measured values (Table 2 ) appear much lower than those displayed by $\mathrm{Pb}$ and $\mathrm{Ra}$ in experiments of adsorption on $\mathrm{MnO}_{2}$ carried out in water for comparable temporal range and "mass adsorbent /volume solution" ratio $[33,48-50]$. The notable difference of adsorption of ${ }^{212} \mathrm{~Pb}$ and ${ }^{228} \mathrm{Ra}$ ions between NAPL (kerosene or hexane) and water suggests that some mechanism hinders the adsorption of cations in apolar liquids allowing to achieve $R_{\text {NAPL real }}$ values close to unity( like in $n$-hexane). Two processes can contribute to this effect. The first is the adsorption of organic polar compounds onto $\mathrm{MnO}_{2}$. This process could partially work for kerosene but not for pure hexane. The second is the phenomenon of electrostriction in apolar liquids [70]. In these fluids, a spherical positive impurity ion extends notably its electrostrictive influence into the liquid environment. The inclusion of a charge in a non-polar medium with a low permittivity has a range effect on any other present charge much longer than in an aqueous medium [71]. According to the Bjerrum theory, ions might exist separately only if their size exceeds the Bjerrum radius [72]. Bjerrum radius is of about $0.7 \mathrm{~nm}$ in water and of $28 \mathrm{~nm}$ in non-polar liquids of low permittivity $(\approx 1.8)$ like kerosene or hexane. Electrostriction causes the formation of a cluster of non-polar species around the ion. It may be expected that the ion exists in a polarized atmosphere of neighbour molecules as a relatively large polarized clump that moves through the liquid [73]. The adjacent layers around the ion are layers of high viscosities which approach the bulk viscosity of the liquid only further away from the ionic core [70]. These layers "hinder" the adsorption of simple ions as Ra on adsorption sites. The 


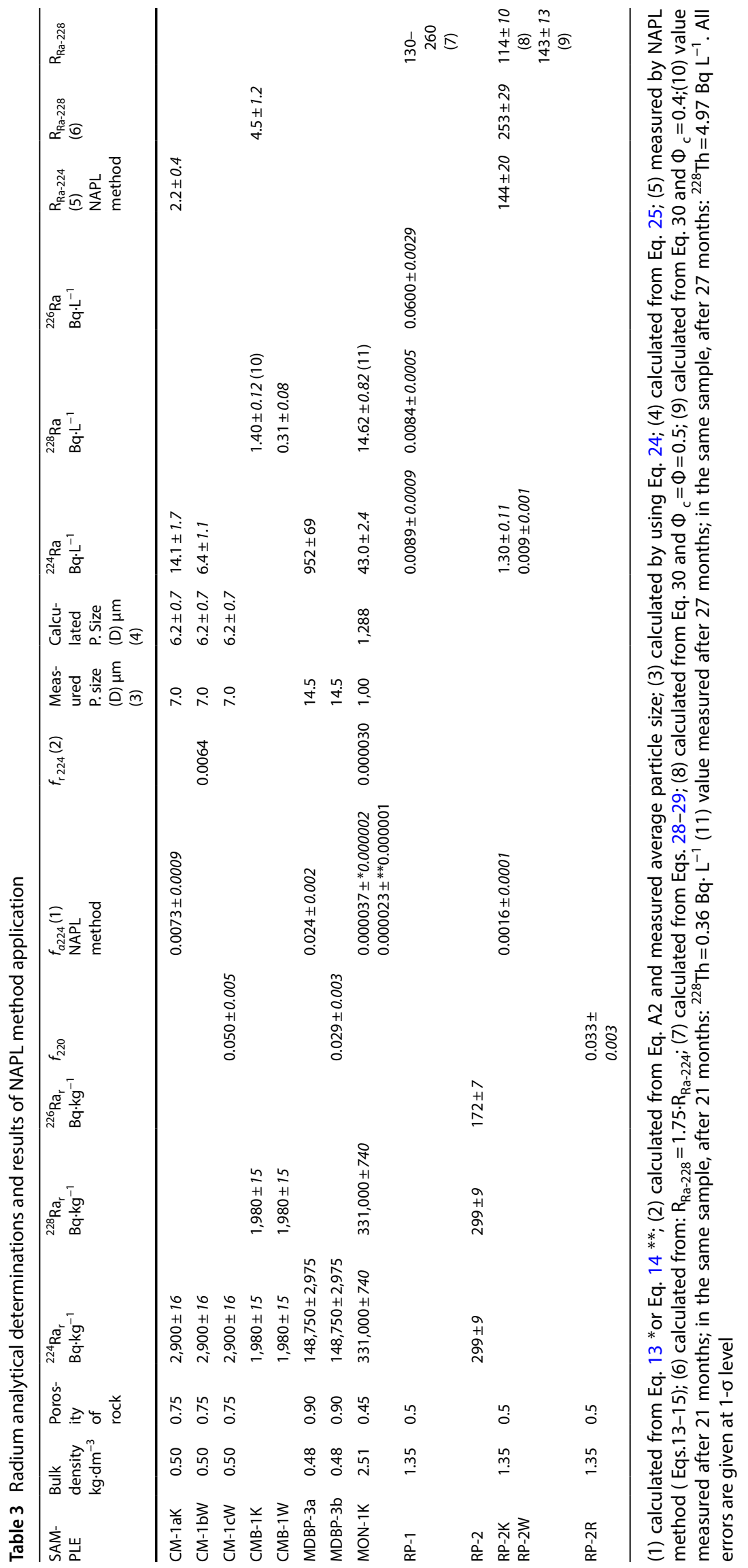


resilient occurrence of Ra adsorption in MDBP- $1 \mathrm{~K}$ is likely due to the exceptional specific surface of $\mathrm{MnO}_{2}$ that even in extreme saline solutions, where the retardation factor of $\mathrm{Ra}$ is close to 1 (as in Dead Sea water), is still capable to adsorb radium [34] with an efficiency up to $15 \%$.

\subsubsection{Experiments MDBP-2}

In sample MDBP-2, ratio of adsorbed ${ }^{228}$ Ra to desorbed ${ }^{228} \mathrm{Ra}$ in kerosene is equal to $1.18 . \mathrm{K}_{\text {dreal }}^{*}$ and $\mathrm{R}_{\text {NAPL real }}$ of ${ }^{228} \mathrm{Ra}$ are $10.5 \pm 0.6$ and $11.5 \pm 0.6$. These values (Table 2 ) show that Ra, when occurs in apolar fluids as a large complexed ion, is moderately adsorbed on $\mathrm{MnO}_{2}$. Therefore, electrostriction is important as adsorption-hindering mechanism only for simple positive $\mathrm{Ra}$ ions being less efficient when Ra occurs as large sized complexed ion.

\subsubsection{Experiments MDPB-3}

Results of this experiment are given on Table 3. The mean diameter of MDBP particles is $\sim 14.5 \mu \mathrm{m}$, when calculated from mode $(7 \mu \mathrm{m})$ and median $(12 \mu \mathrm{m})$ of the size (diameter) distribution. For asymmetric distribution, the three statistics are connected by the empirical relation [74]:

Mean $=\frac{[(3 \cdot \text { Median })-\text { Mode }]}{2}$.

In MDBP-3 experiment, initial Ra occurs exclusively as adsorbed ion on the external surface of the sample. In this case, the model of Maraziotis (Eqs.35-37) does not apply, the theoretical fraction from a-recoil being given by Eq. 4. The $f_{a 224}$ of MDBP-3a, obtained by the NAPL method (Eq. 14), is equal to $24 \pm 2 \cdot 10^{-3}$. The mean of 12 measurements of ${ }^{220} \mathrm{Rn}$ emanation coefficient, $f_{220}$, (MDBP-3b) is similar: $29 \pm 3 \cdot 10^{-3}$. In case of $\mathrm{R}_{\text {Ra224 }}=1$, in this particular experiment, $f_{a 224}$ and $f_{220}$ are expected to be quite similar (apart a difference of $5 \%$ due to their $Q_{a^{\prime}}$ the a-decay energy of their parent atoms). Their difference is of $20 \%$, corresponding to a $R_{\text {Ra224 }}$ of $1.21 \pm 0.16$ which is very close, taking in account the associated error, to the assumed value of $\mathrm{R}_{\mathrm{Ra} 224}=1$ in NAPL. The low absolute values of $f_{a 224}$ and $f_{220}$ (compared with the theoretical value of 0.50 in absence of embedding effects) are likely due to embedding phenomena [26, 27]. The consistence of the embedding model can be tested by applying Eq. 4 and by comparing the obtained $f_{a 224}$ with $f_{220}$. By considering the recoil energy of ${ }^{224} \mathrm{Ra}$ and its $\mathrm{R}_{\mathrm{F}}$ in kerosene (similar to polybutadiene [75]), it obtains a pore size $(w)$ of $5.2 \mathrm{~nm}$. A comparable pore size $(5.3 \mathrm{~nm})$ is calculated by considering the recoil energy of ${ }^{220} \mathrm{Rn}$ and its $\mathrm{R}_{\mathrm{F}}$ in water. This pore size matches that observed in synthetic and commercial $\mathrm{MnO}_{2}$ $[76,77]$. This experiment shows that only if Ra is exclusively located in grains as adsorbed ion, it enters the liquid phase by the same mechanism of radon (direct recoil) and the two methods, NAPL and radon comparison, coincide.

\subsection{Ra adsorption on zeolite $4 \mathrm{~A}$}

In Z4A-1, the ratio of adsorbed ${ }^{224} \mathrm{Ra}$ to desorbed ${ }^{224} \mathrm{Ra}$, $\mathrm{K}_{\text {dreal }}^{*}$ and $\mathrm{R}_{\text {NAPL real }}$ in kerosene are equal to $0.02 \pm 0.03$, $0.04 \pm 0.07$ and $1.04 \pm 0.07$, respectively. These results (Table 2) confirm the assumption that in NAPL the retardation factor of Ra simple ions is close to unity. Differently in Z4A-2 (Ra complex ion in kerosene) and especially in Z4A-3 (Ra simple ion in water), the real Ra retardation factors are higher $(2.19 \pm 0.07$ and $16.0 \pm 2.3$, respectively). The formation of clusters of molecules of apolar fluid around simple Ra ions, induced by electrostriction, explains this behaviour. They prevent Ra adsorption on elite in kerosene especially when the electrostrictive effect of the Ra charge is not lowered by formation of Ra-complexes with large organic molecules. As large complexed ion (Z4A-2), instead, $\mathrm{Ra}$ is adsorbed significantly on zeolite (like on MDBP) in apolar liquids. TTA-TOPO-Ra complexes, together to the crown ether-Ra complexes, are the only ones known to be soluble in apolar liquids but both are not present in common NAPLs. Thus, the suggestion of using common apolar liquids like kerosene to determine $R_{R a}$ is strengthen.

\subsection{Ra adsorption on clay}

As expected, in S8N1-3 W the real Ra retardation factor in water is high (23.2 \pm 2.2 ) (Table 3). Differently, in S8N1-1 K and S8N1-3 K the $R_{\text {NAPL real }}$ are close to unity $(1.27 \pm 0.18$, $1.27 \pm 0.27$, respectively). Clay minerals have the highest capacity of adsorbing NAPLs between common minerals [78]. By comparing Ra in NAPL (S8N1-1 K, S8N-3 K) and in water (S8N1-3 W), it can be concluded that NAPL molecules, wetting clay surfaces, hinder the development of double layer reducing the adsorption of inorganic ions as yet suggested [79]. Clay minerals are ubiquitous in soil and $\mathrm{Ra}$ isotopes seem to be unaffected by adsorption when clay is immersed in NAPL; therefore, the determination of the contact time between NAPLs and particle grains of sediments or soils is possible, as the following two sections will show.

\subsection{Monazite in contact with NAPL}

The surface of monazite grains is charged negatively and adsorbs positive ions as $\mathrm{Ce}$, $\mathrm{La}$ and $\mathrm{Ca}$ [80]. In particular, adsorption of divalent elements like $\mathrm{Ca}, \mathrm{Ba}$ and $\mathrm{Ra}$, is high when they occur as $\mathrm{Ca}(\mathrm{Ra}, \mathrm{Ba}) \mathrm{OH}^{+}$, via hydrogen bonding with phosphate-oxygen active sites negatively charged on the monazite surface $[81,82]$. The $f_{a 224}$ of MON-1 K (NAPL 


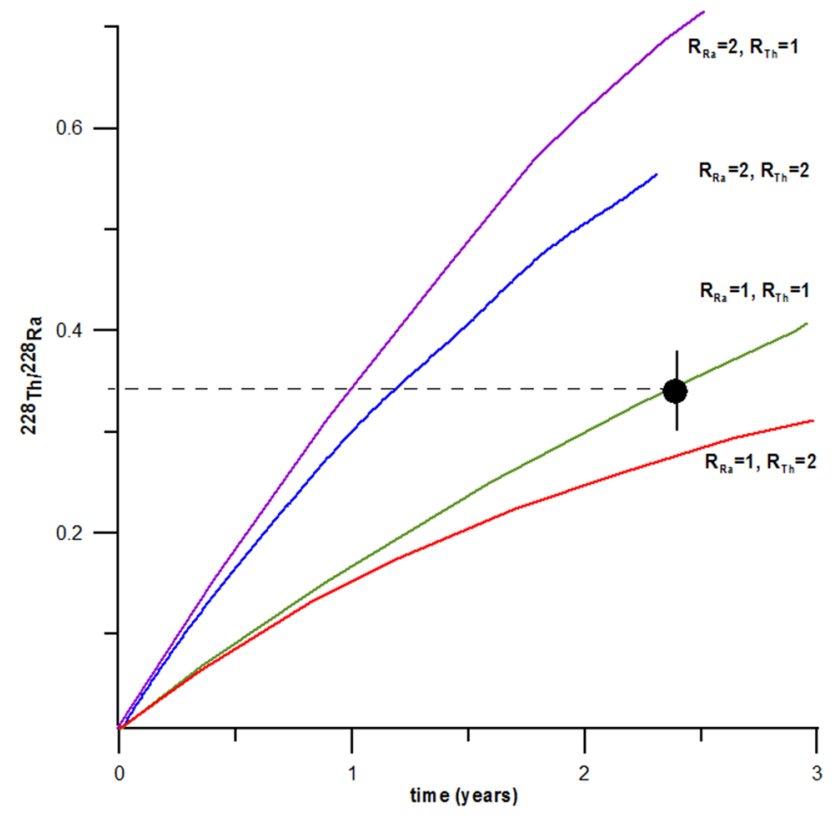

Fig. 6 Evolution of ${ }^{228} \mathrm{Th} /{ }^{228}$ Ra activity ratio in kerosene according to Eq. 21 for different couples of Th and Ra retardation factors. Full black circle: ${ }^{228} \mathrm{Th} /{ }^{228} \mathrm{Ra}$ activity ratio in kerosene after 27 months of contact with monazite

method, Table 3) calculated by Eq. $13\left(3.7 \cdot 10^{-5}\right)$ or Eq. 14 $\left(2.3 \cdot 10^{-5}\right)$, is comparable to the $f_{\mathrm{r}}\left(3.0 \cdot 10^{-5}\right)$ resulting from Eq. 35 , therefore a-recoiled $\mathrm{Ra}$ is not adsorbed onto monazite in apolar fluid.

The ${ }^{228} \mathrm{Th} /{ }^{228}$ Ra activity ratio in kerosene, measured after 27 months of contact between kerosene and monazite, is equal to $0.34 \pm 0.4$ corresponding to a time of contact of $28 \pm 3.5$ months.

Figure 6 shows the good matching between ${ }^{228} \mathrm{Th} /{ }^{228} \mathrm{Ra}$ activity ratio measured in kerosene and its theoretical value after 27 months of contact between kerosene and monazite. This is a clear evidence, as mentioned before, that retardation factors of Ra and Th are both close to 1 in kerosene. The temporal evolution of the ${ }^{228} \mathrm{Th} /{ }^{228} \mathrm{Ra}$ activity ratio is sensible to the values of the retardation factors. Thus, this correspondence is a strong argument in favour of inhibition of the adsorption of inorganic positive ions on charged sites of mineral surfaces in contact with apolar fluids. The results suggest that this radioactive pair can be useful for dating NAPL pollution in sediments or soil which contain always some ${ }^{232} \mathrm{Th}$ that generates ${ }^{228}$ Ra recoiled atoms into NAPL in contact with them [83].

\subsection{Application of the NAPL method for determining retardation factor and recoil constant of Ra for a Th-enriched weathered volcanic rock}

The ${ }^{228} \mathrm{Th} /{ }^{228}$ Ra activity ratio in CMB- $1 \mathrm{~K}$, measured after 21 months of contact between kerosene and monazite, is equal to $0.26 \pm 0.4$ corresponding to a time of contact of $21 \pm 4$ months (Table 3 ). As before, this results confirms that retardation factors of Ra and Th are both close to 1 in kerosene and the possibility to use this radioactive pair to estimate the time of contact between NAPL and soil.

The mean particle diameter of $\mathrm{CM}-1$ was calculated (Eq. 29) from mode $(40 \mu \mathrm{m})$ and median $(18 \mu \mathrm{m})$ obtaining a value of $7.0 \mu \mathrm{m}$. The $f_{a 224}$ of the CM-1 aK is equal to $7.3 \pm 0.9 \cdot 10^{-3}$ (Table 3). Remembering that the intraparticle pore recoil component of Ra can be neglected then $f_{\alpha}=f_{\mathrm{r}}$ and combining Eq. 13 and Eq. 35 it obtains:

$f_{\mathrm{r} 224}=f_{\alpha 224}=\frac{{ }^{224} \mathrm{Ra}_{\mathrm{NAPL}}}{{ }^{224} \mathrm{Ra}_{r}} \cdot \frac{\Phi}{\rho_{r}}=\frac{3 \mathrm{R}_{\mathrm{c}}}{4 \alpha}-\frac{1}{16} \cdot\left(\frac{\mathrm{R}_{\mathrm{c}}}{\alpha}\right)^{3}$

From Eq. 25 , knowing $f_{a 224}$, measured by NAPL method, and $\mathrm{R}_{\mathrm{c}}$ in the particle, the average particle diameter (2a) has been estimated $(6.2 \mu \mathrm{m})$ resulting comparable with the value found by laser granulometry. By using the mean diameter of $7.0 \mu \mathrm{m}, f_{\mathrm{r} 224}$ of $\mathrm{CM}-1$ is equal to $6.4 \cdot 10^{-3}$, a value fitting the measured one $\left(f_{a 224}\right.$ of $\left.\mathrm{CM}-1 \mathrm{aK}=7.3 \cdot 10^{-3}\right)$. The intraparticle pore recoil component $\left(f_{\mathrm{p} 224}=f_{a 224}\right.$ $f_{\text {r224 }}=0.9 \cdot 10^{-3}$ ) is clearly trascurable. This is not the case for ${ }^{220} \mathrm{Rn}$. The average of 12 measurements of $f_{220}(\mathrm{CM}-1 \mathrm{cW})$ is in fact equal to $50 \pm 5 \cdot 10^{-3}$. Therefore by subtracting the sum of $f_{\mathrm{r} 220}$ and $f_{\mathrm{d} 220}$ to $f_{220}$, a relevant ${ }^{220} \mathrm{Rn}$ intraparticle pore recoil component, $f_{\mathrm{p} 220}=43 \cdot 10^{-3}$, is obtained. Thus the "Rn method" should give a $\mathrm{R}_{\mathrm{Ra}-224}$ seven times higher than that obtained by "NAPL method". This difference depends on $f_{\mathrm{p}}$ : it is negligible for ${ }^{224} \mathrm{Ra}$ but not for ${ }^{220} \mathrm{Rn}$. Nanobubbles of air occur commonly in solid-state nanopores immersed in liquids [84]. Liquids in nanopores of silicates, moreover, do not form a compact structure of connected molecules but rather a collection of small clusters forming plugs separated by air bubbles [85]. Thus, any nanometric air bubble along the path produces a fast electrostatic attachment of $\mathrm{Ra}$ ions on the walls of the pore, reducing notably $f_{\mathrm{p} 224}$. Differently, $\mathrm{Rn}$ atoms can continue to diffuse in the pore. The $\mathrm{R}_{\mathrm{Ra}-224}$ obtained by dividing the ${ }^{224} \mathrm{Ra}$ content of $\mathrm{CM}-1 \mathrm{aK}$ to that of $\mathrm{CM}-1 \mathrm{bW}$, and the $\mathrm{R}_{\mathrm{Ra}-228}$, obtained by dividing the ${ }^{228} \mathrm{Ra}$ content of CMB-1 $\mathrm{K}$ to that of CMB- $1 \mathrm{~W}$, are equal to $2.2 \pm 0.4$ and $4.5 \pm 1.2$, respectively. The ratio $R_{R a-228 /} R_{R a-224}=2.04 \pm 0.64$ is therefore comparable with the mean value of 1.75 found from literature data. Finally, the relatively low $R_{R a} s$ are likely due to 


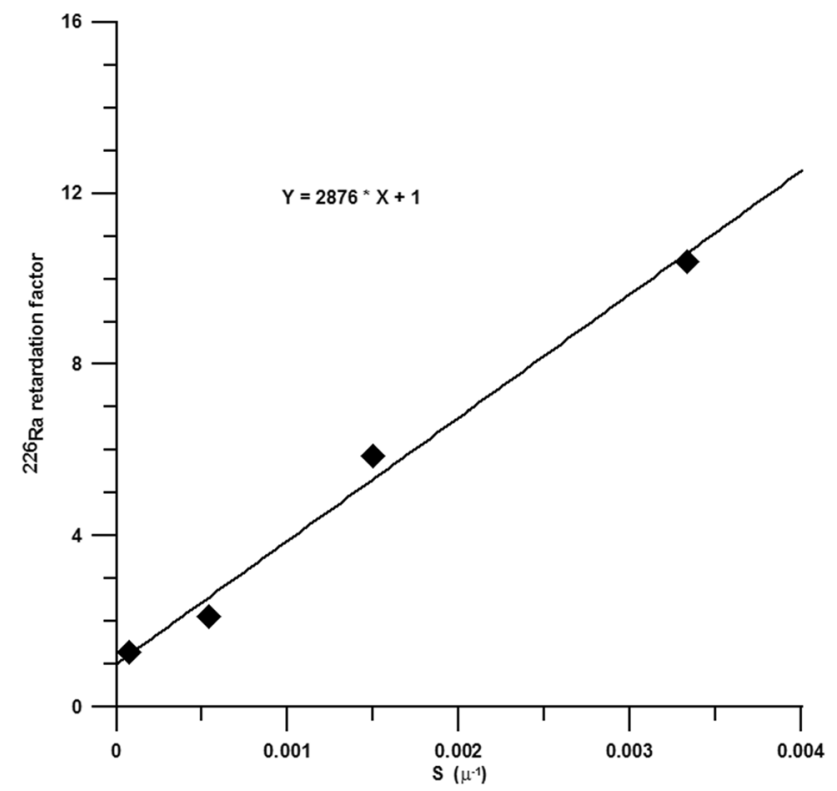

Fig. 7 Variation of ${ }^{226}$ Ra retardation factor due to leucite versus S. The ${ }^{226} \mathrm{Ra}$ retardation factor is calculated from experimental values of $\mathrm{K}_{\mathrm{A}}$ and $\mathrm{S}$

the complete obliteration of leucite (a Ra-adsorber phase causing high retardation factors) by weathering processes.

\subsection{Ra adsorption onto leucite}

Figure 7 reports ${ }^{226}$ Ra adsorption data on leucite. The TSA (total surface area) $/ V_{S}$ (volume solution) ratio is plotted vs. the $\mathrm{R}_{\mathrm{Ra}-226}$ calculated adopting the Sugita-Gillham formalism [86]. According to it, $R_{\mathrm{Ra}-226}$ depends on the product of the distribution coefficient $\mathrm{K}_{\mathrm{A}}$ defined on an area basis $\left(\mu \mathrm{m}^{-1}\right)$ and the TSA $/ \mathrm{V}_{\mathrm{S}}$ ratio:

$\mathrm{R}_{\mathrm{Ra}-226}=1+\left(\frac{\mathrm{K}_{A} \cdot \mathrm{TSA}}{\mathrm{V}_{S}}\right)=1+\left(\mathrm{K}_{A} \cdot \mathrm{S}\right)$

The TSA $/ \mathrm{V}_{\mathrm{S}}$ ratio equals $\mathrm{S}$, the extent of the rock surface in contact with unit volume of groundwater $\left(\mu \mathrm{m}^{-1}\right)$. The distribution coefficient between adsorbed phase and solution, $\mathrm{K}_{\mathrm{A}}$, defined on an area basis, is equal to:

$\mathrm{K}_{A}=\frac{{ }^{226} \mathrm{Ra} \text { activity per unit surface area }}{{ }^{226} \text { Ra activity per unit volume of solution }}$.

The $\mathrm{R}_{\mathrm{Ra}-226}$ due to adsorption on leucite, obtained by linear best-fitting through the plotted points, is:

$\mathrm{R}_{\mathrm{Ra}-226}=1+\left(\frac{2.88 \cdot 10^{3} \cdot \mathrm{TSA}}{\mathrm{V}_{S}}\right)$

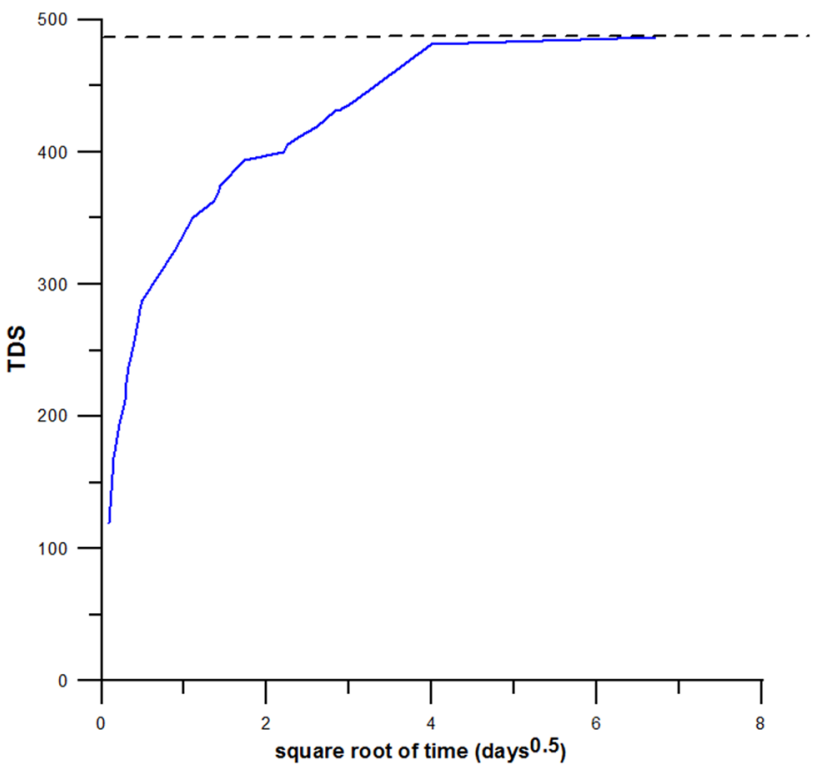

Fig. 8 Initial dissolution of Red Pozzolane in distilled water at $\mathrm{pH}$ 6.0-6.5: Recorded TDS values over time. Dotted line signs the limit value of $475 \mathrm{mg} \mathrm{L}^{-1}$

In order to find the ratio TSA $/ \mathrm{V}_{\mathrm{S}}$, which equals $\mathrm{S}$, the Carman-Kozeny equation for porous strata and spherical particle grains was used [87]:

$S\left(\mu m^{-1}\right)=\left\{\frac{\left[\frac{\Phi^{3}}{(1-\Phi)^{2}}\right]}{k \cdot 5}\right\}^{0.5}$

where $k$ is the mean permeability coefficient.

By knowing that $\Phi=0.50$ and $k=5 \cdot 10^{-1} \mu \mathrm{m}^{2}$ in $\operatorname{RP}[88$, 89], a value for $S$ of $0.45 \cdot \mathrm{m}^{-1}$ is obtained. This value, multiplied for the modal fraction of leucite in RP (0.1-0.2), gives a $\mathrm{R}_{\mathrm{Ra}-226}$ due to leucite in the range of 130-260. The implications of this result are discussed in the following section.

\subsection{Study site: Ra isotopes in groundwater from the Red Pozzolane aquifer (Alban Hills)}

Results of analytical determination of radium isotopes in RP groundwater are given in Table 3. The Ra isotopic composition of RP-1 is equal to $8.9 \pm 0.9 \cdot 10^{-3} \mathrm{~Bq} \cdot \mathrm{L}^{-1}$ for ${ }^{224} \mathrm{Ra}$, $8.4 \pm 0.5 \cdot 10^{-3} \mathrm{~Bq} \cdot \mathrm{L}^{-1}$ for ${ }^{228} \mathrm{Ra}$ and $60.0 \pm 2.9 \cdot 10^{-3} \mathrm{~Bq} \cdot \mathrm{L}^{-1}$ for ${ }^{226} \mathrm{Ra}$. The $f_{a 224}$ of RP- $2 \mathrm{~K}$, obtained by the NAPL method, results equal to $1.6 \pm 0.1 \cdot 10^{-3}$. The $R_{\text {Ra-224, }}$ calculated from RP- $2 \mathrm{~K}$ and RP-2 W, by using the NAPL method (Table 3 ), is close to $144 \pm 20$. The average of 12 measurements of the ${ }^{220} \mathrm{Rn}$ emanation coefficient, $f_{220}$, carried out on RP-2R, results equal to $33 \pm 3 \cdot 10^{-3}$. Figure 8 , where TDS is plotted 
vs. $T^{0.5}$, shows the variation of initial dissolution of RP in groundwater. At the end of the initial dissolution ( 45 days), TDS trends to a stationary value of $487 \mathrm{mg} \cdot \mathrm{I}^{-1}$, close (about $85 \%)$ to that measured in the field $\left(572 \mathrm{mg} \cdot \mathrm{I}^{-1}\right)$. The increase of $\mathrm{pH}$, at the end of experiment, is $<0.5 \mathrm{pH}$ units $\left(\mathrm{pH}_{\text {initial }}=6\right) \cdot{ }^{226} \mathrm{Ra}_{w(0)}$ and ${ }^{228} \mathrm{Ra}{ }_{w(0)}$, then, are calculated according to:

${ }^{226} \mathrm{Ra}_{w}(0)\left(\mathrm{Bq} \cdot \mathrm{L}^{-1}\right)=$ TDS stationary $\left(\mathrm{kg} \cdot \mathrm{L}^{-1}\right) \cdot{ }^{226} \mathrm{Ra}_{\mathrm{r}}$ $\left(\mathrm{Bq} \cdot \mathrm{kg}^{-1}\right)=0.487 \cdot 10^{-3} \cdot 172=0.084 \mathrm{~Bq} \cdot \mathrm{L}^{-1}$,

${ }^{228} \mathrm{Ra}_{w(0)}\left(\mathrm{Bq} \cdot \mathrm{L}^{-1}\right)=$ TDS stationary $\left(\mathrm{kg} \cdot \mathrm{L}^{-1}\right) \cdot{ }^{228} \mathrm{Ra}_{\mathrm{r}}(\mathrm{Bq} \cdot \mathrm{k}$ $\left.\mathrm{g}^{-1}\right)=0.487 \cdot 10^{-3} \cdot 299=0.146 \mathrm{~Bq} \cdot \mathrm{L}^{-1}$.

A first estimation of $R_{\mathrm{Ra}-228}$ and $\mathrm{R}_{\mathrm{Ra}-226}$ was done by

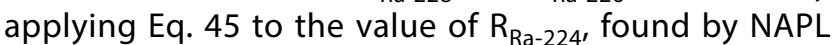
method (see Appendix 5). It gives a value of $253 \pm 29$, in the range of the values estimated for the only leucite which plays, in this aquifer, an important role in Ra adsorption. In $\mathrm{RP}$, the contribute of the initial dissolution of aquifer rock to Ra activity cannot be neglected, as yet others evidenced [3]. Thus, Eqs. 50-52 (see Appendix 5) were applied. Since the first estimate of $\mathrm{R}_{\mathrm{Ra}-228}$ is very high, exponential terms can be considered negligible and Eq. 50 becomes:

${ }^{228} \mathrm{Ra}_{w}=\left[\frac{f_{\alpha 224} \cdot 0.74}{\mathrm{R}_{R a-228}}\right] \cdot\left({ }^{228} \mathrm{Ra}_{r} \cdot \frac{\rho_{r}}{\Phi}\right)$

The last equation, known $f_{a 224}$ (from NAPL method) and the other variables $\left(\Phi, \rho_{r}{ }^{228} R a_{r}\right)$ of the aquifer, gives a second estimate of $\mathrm{R}_{\mathrm{Ra}-228}=\mathrm{R}_{\mathrm{Ra}-226}=114 \pm 10$. This second estimate, lower than the value of $R_{\text {Ra-224 }}(144 \pm 20)$ found with the NAPL method, seems to contradict the previous statement that the $\mathrm{R}_{\mathrm{Ra}-228}$ / $\mathrm{R}_{\mathrm{Ra}-224}$ ratio is $\geq 1$. Indeed up to now it has been implicitly assumed that porosity in porous aquifers corresponds to the pore connectivity. However this is not true especially for vesiculated volcanic aquifer rocks. In this case $\Phi$ in Eq. 36 should be substituted by the pore connectivity $\Phi_{c}$ which is the product of $\Phi$ and connectivity C [90]. The variable vesicularity of Red Pozzolane [42], from poorly to moderate (0-20\%), suggests that pore connectivity could reach the value of 0.40 . In this case, the contradiction should be solved because Eq. 36 yields $R_{\text {Ra-228 }}=R_{\text {Ra-226 }}=143 \pm 13$. Anyway, after averaging the two extreme values (114 and 253), we assume $184 \pm 15$ as the more reliable value for $\mathrm{R}_{\mathrm{Ra}-228}=\mathrm{R}_{\mathrm{Ra}-226}$. According to Eq. 52, the age of groundwater sampled at the Arco di Travertino site results equal to $5.5 \pm 0.4$ years. This is the age of the groundwater at the sampled depth which, however, differs from the mean residence time, MRT. A critical problem in MRT determination, in fact, is the dispersion of groundwater age, i.e. the relationship between MRT and the depth of aquifer below the water table. Age increases with the depth of sampling respect to the level of water table, and the values can be spread by a factor 50 [91]. It is

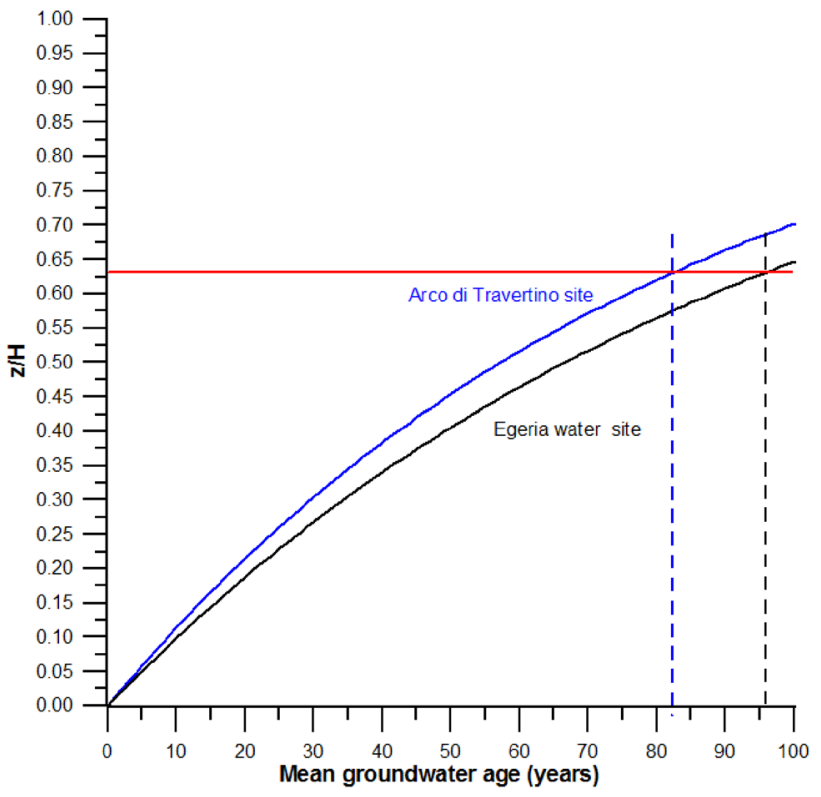

Fig. 9 Mean residence time of Alban Hills groundwater at the Arco di Travertino site and at Egeria mineral water site, calculated from Eqs.32-33

however possible to use an empirical equation to correlate the recharge rate $R$ to the groundwater age [92]:

$R=\left\{\ln \left[\frac{H}{(H-z)}\right]\right\} \cdot\left(\frac{H \cdot \Phi}{T}\right)$

where $R$ is the recharge rate $\left(\mathrm{m} \mathrm{y}^{-1}\right), T$ the groundwater age, $H$ the height of the water column and $z$ is the distance from the sampling point and the upper level of the water table. Equation 31 can be expressed as well as:

$\frac{Z}{H}=1-\mathrm{e}^{-\frac{R \cdot T}{H \cdot \Phi}}$

When this last equation is rendered graphically, the MRT is given by the intersection point of $z / \mathrm{H}$ function with the line corresponding to $z / H=1-\mathrm{e}^{-1}=0.632$ (Fig. 9). In the case of the piezometric well at Arco di Travertino (Fig. 5) $\mathrm{H}=23, \mathrm{z}=1.5, \Phi=0.5$ and $T=5.5 \pm 0.4 \mathrm{y}$, therefore $R=0.141 \pm 0.011 \mathrm{~m} \mathrm{y}^{-1}$. This value matches that estimated by others $\left(0.152 \mathrm{~m} \cdot \mathrm{y}^{-1}\right)$ for the same hydrogeological basin [89].

In this exponential age distribution, the mean residence time (MTR) is given by [92]:

$\mathrm{MTR}=H \cdot \Phi / R$.

Applying this equation to the site of Arco di Travertino, a MTR of $82 \pm 6$ years is obtained. Of course, establishing the exact average residence time of groundwater of the entire Alban Hills aquifer is beyond the scope of this work. 
This example was made to emphasize that the estimated local MRT, even for a single well, is realistic when using the NAPL method. Differently, by using the ${ }^{220} \mathrm{Rn} /{ }^{224} \mathrm{Ra}$ ratio for estimating the retardation factor of ${ }^{224} \mathrm{Ra}$, a value of 3000 is obtained, $\sim 21$ times $\left(f_{220} / f_{a 224} \sim 21\right)$ higher than that measured by NAPL method and, consequently, an unrealistic low MRT (3.9 years). The goodness of the model (Eq. 52 and Eq. 32) can be verified comparing these results to the age and MRT of the Egeria mineral water, springing at about $1 \mathrm{~km}$ at SE of the Arco di Travertino site, inside the same RP aquifer. At Egeria site, the presence of a $\mathrm{CO}_{2}$ bubble plume has likely destructed the age stratification of groundwater [93] (Fig. 5). Therefore, in this site, the groundwater age T coincides with MRT. From available hydrogeological maps and geological profiles $[42,94]$ the thickness of the aquifer is, at Egeria site, of $27 \mathrm{~m}$. By assuming the calculated $R$ of $0.141 \pm 0.011 \mathrm{~m} \mathrm{y}^{-1}$, a MRT of $96 \pm 8$ years is obtained from Eq. 33 (Fig. 9). It is interesting to compare this predicted value with the one resulting by applying Eq. 50-52 (see Appendix 5) to Ra in groundwater for this site. Available data on ${ }^{226} \mathrm{Ra}$ and ${ }^{228} \mathrm{Ra}$ for the Egeria mineral water are equal to $3.56 \cdot 10^{-3}$ and $10.2 \cdot 10^{-3} \mathrm{~Bq} \cdot \mathrm{L}^{-1}$, respectively [95]. Assuming the same $\mathrm{R}_{\mathrm{Ra}-226}(184 \pm 15)$ and ${ }^{226} \mathrm{Ra}_{w(0)}\left(0.084 \mathrm{~Bq} \cdot \mathrm{L}^{-1}\right)$ found for the Arco di Travertino site, it obtains a value of $84 \pm 7$ years, quite comparable with the predicted one.

\section{Conclusions}

Adsorption experiments on Ra-adsorbing materials (manganese dioxide, zeolite, clay,) immersed in NAPL and measurements of the time of contact NAPL /monazite and NAPL/weathered volcanic rock by ${ }^{228} \mathrm{Th} /{ }^{228} \mathrm{Ra}$ activity ratio have been carried out. The experiments suggest that when these materials are immersed in non-aqueous phase liquids (NAPL) adsorption on particle grains is strongly reduced (as in the case of manganese dioxide) or absent (as in the case of the other tested materials). This is evident especially when distribution coefficient is corrected for the particle concentration effect. A method based on this observation, the NAPL method, has been described and applied to estimate recoil rate constant and retardation factor of Ra isotopes. When retardation factor and recoil rate constant of Ra isotopes are estimated by the NAPL method, the obtained values result always lower than those found using radon as comparing term. Indeed radon is introduced in solution by a more complex way than radium. Radium is introduced essentially by direct a-recoil, whereas radon by further mechanisms as back diffusion. The NAPL method is based on the theory of electrostriction which predicts that, in nonpolar solutions, simple ions are surrounded by non-polar molecules. These molecules are feebly polarized by the electrical field of ions occurring in non-polar fluids. The polarization power of these ions, in non-polar fluids, is about 30 times stronger than in polar fluids like water. At the interface between charged surface of adsorbent and NAPL, the same electrostrictive phenomena allow also a full saturation of adsorption sites by polar and non-polar molecules of NAPL. The result of the two effects is a negligible amount of adsorbed a-recoiled nuclides onto the surface of particle grains and an increase in the conservative behaviour of these radionuclides in NAPL in comparison with water. By knowing retardation factor and recoil constant of radium through NAPL method, the equations describing the evolution of radium isotopes in aquifers can be solved in many cases. An example is the determination of residence time in upper shallow aquifer of Alban Hills mainly hosted in Red Pozzolane units. An interesting implication of the results of this paper is also the theoretical possibility to date the NAPL contamination time of soils, from medium ( $>10 \mathrm{ppm}$ ) to high content of thorium, by measuring the evolution of the ${ }^{228} \mathrm{Th} /{ }^{228} \mathrm{Ra}$ ratio in NAPLs [83].

Author contributions All authors have equally contributed to the laboratory work, theoretical elaboration and writing of this research paper. All authors read and approved the final manuscript.

Funding Only institutional fundings of IGAG-CNR and of Università Roma TRE were involved in the preparation of this research paper.

Availability of data and material The main data set of experiments is given in the paper in Table 1. Gamma spectra of analysed sample are stored and saved at IGAG -CNR Environmental Geochemistry Laboratory.

\section{Compliance with ethical standards}

Conflict of interest No competing interests among authors or between CNR and Università Roma TRE are involved in the preparation of this research paper.

\section{Appendix 1}

\section{Derivation of the master equation}

According Kiro et al. [6, 7], by assuming one-dimensional steady-state flow coupled with negligible hydrodinamic dispersion and negligible co-precipitation, the solution of the differential equation describing the temporal variation of Ra activity in groundwater along a single flow path $x$ with constant velocity $v_{x}$ and constant $\mathrm{K}_{d}^{*}$ is 
${ }^{i} \operatorname{Ra}_{w}=\left[\frac{\mathrm{P}}{\lambda_{i} \cdot\left(\mathrm{K}_{\mathrm{d}}^{*}+1\right)}\right] \cdot\left(1-\mathrm{e}^{-\lambda_{i} \cdot\left(\mathrm{K}_{\mathrm{d}}^{*}+1\right) \cdot\left(\frac{x}{v_{x}}\right)}\right)+{ }^{i} \mathrm{Ra}_{w(0)} \cdot \mathrm{e}^{-\lambda_{i} \cdot\left(\mathrm{K}_{\mathrm{d}}^{*}+1\right) \cdot\left(\frac{x}{v_{x}}\right)}$

According Kiro et al. [7], $\mathrm{P}$ is the production rate of ${ }^{i} \mathrm{Ra}_{w}$ activity $\left(\mathrm{Bq} \cdot \mathrm{t}^{-1}\right)$ from the aquifer solids by recoil and dissolution and it is given by:

$\mathrm{P}=\left(\Delta_{i}+\Gamma_{i}\right) \cdot{ }^{i} \mathrm{Th}_{r} \cdot \frac{\rho_{r}}{\Phi}$.

Firstly, in case of radioactive equilibrium in the aquifer rock, ${ }^{i} \mathrm{Th}_{r}={ }^{i} \mathrm{Ra}_{r}$; then ii) $\left(\frac{x}{v_{x}}\right)$, the ratio between the length of the linear radionuclide path and its velocity, is equal to $\mathrm{T}$; finally, iii) $\left(\mathrm{K}_{\mathrm{d}}^{*}+1\right)=\mathrm{R}_{i}$.

Therefore, Eq. 1 is easily obtained: coefficient of $\mathrm{Rn}$ in feldspar at the same temperature is tenth orders higher, about $10^{-31} \mathrm{~m}^{2 \mathrm{~s}-1}[12]$.

\section{Appendix 4}

\section{Limits of variation of the $\mathrm{R}_{\mathrm{Ra}-228} / \mathbf{R}_{\mathrm{Ra}-224}$ ratio}

A recent paper [35] assumes $R_{228} / R_{224}=1$ and desorption rate constant $k_{d}>>\lambda_{224}\left(0.19\right.$ day $\left.^{-1}\right)$. Other authors, more

${ }^{i} \operatorname{Ra}_{w}=\left[\frac{\left(\Delta_{i}+\Gamma_{i}\right)}{\left(\lambda_{i} \cdot \mathrm{R}_{i}\right)}\right] \cdot\left(1-\mathrm{e}^{-\lambda_{i} \cdot \mathrm{R}_{i} \cdot \mathrm{T}}\right) \cdot\left({ }^{i} \operatorname{Ra}_{r} \cdot \frac{\rho_{r}}{\Phi}\right)+{ }^{i} \operatorname{Ra}_{w(0)} \cdot\left(\mathrm{e}^{-\lambda_{i} \cdot \mathrm{R}_{i} \cdot \mathrm{T}}\right)$

\section{Appendix 2}

\section{Components of $R \mathbf{n}$ and Ra emanation}

They are defined by [13]:

$f_{r}=\frac{3 R_{c}}{4 \alpha}-\frac{1}{16} \cdot\left(\frac{R_{c}}{\alpha}\right)^{3}$

$f_{p}=\frac{R_{c} \cdot k(1+k)}{2 r_{p}}-\frac{1}{16} \cdot\left(\frac{R_{c}}{\alpha}\right)^{3}$

$f_{\mathrm{d}}=\frac{3}{\alpha} \cdot\left(1-f_{\mathrm{r}}-f_{\mathrm{p}}\right) \cdot \sqrt{\frac{\mathrm{D}}{\lambda}}$

where $R_{c}$ is the recoil range in the particle $(\mathrm{nm}), a$ the particle radius $(\mathrm{nm})$ and $k$ its porosity, $r_{p}$ the radius of the nanopores in the particle $(\mathrm{nm}), \mathrm{D}$ the intraparticle diffusion coefficient $\left(\mathrm{nm}^{2} \mathrm{~s}^{-1}\right)$ and $\lambda$ the $\mathrm{Rn}$ decay constant $\left(\mathrm{s}^{-1}\right)$.

\section{Appendix 3}

\section{Diffusion coefficient of $\mathrm{Rn}$ and $\mathrm{Ra}$ in minerals}

No data are available in the literature for diffusion coefficient of radium in minerals at ambient temperature $\left(298^{\circ} \mathrm{K}\right)$. By using data on barium [96], which can be considered the stable analogue of radium for the same ionic charge and similar ionic radius, the Ra diffusion coefficient in feldspar (a common rock-forming mineral), at ambient temperature, should be close to $10^{-80} \mathrm{~m}^{2 \mathrm{~s}-1}$. Diffusion realistically, found that $k_{d}$ values are not less than 0.5 . $\lambda_{224}[4,97,98]$. Thus, by considering that $\lambda_{228}<<k_{d}$, from Eq. 11:

$\left(\frac{\mathrm{R}_{\mathrm{Ra}-228}-1}{\mathrm{R}_{\mathrm{Ra}-224}-1}\right)=\frac{\left(\mathrm{k}_{d}+\lambda_{224}\right)}{\mathrm{k}_{d}}=1+\frac{\lambda_{224}}{\mathrm{k}_{d}}$

By constraining the value of $k_{d}$ to $\geq 0.5 \lambda_{224}$, then:

$3 \geq\left(\frac{\mathrm{R}_{\mathrm{Ra}-228}-1}{\mathrm{R}_{\mathrm{Ra}-224}-1}\right) \geq 1$

or:

$3-\frac{2}{R_{R a}-224} \geq \frac{R_{R a}-228}{R_{R a}-224} \geq 1$

$R_{\text {Ra-224 }}$ is by definition $\geq 1$, then the $R_{\text {Ra-228 }} / R_{\text {Ra-224 }}$ ratio must vary in a narrow range, between 1 and 3 .

\section{Appendix 5}

Assuming dissolution rate constants of ${ }^{228} \mathrm{Ra}$ and ${ }^{226} \mathrm{Ra}$ be equal (congruent dissolution), three typical different cases for Eqs.17-19 are possible:

(1) initial activity of ${ }^{228} \mathrm{Ra}$ and ${ }^{226} \mathrm{Ra}$ can be neglected / a-recoil prevails over dissolution, (2) initial activity of ${ }^{228} \mathrm{Ra}$ and ${ }^{226} \mathrm{Ra}$ can be neglected/ dissolution prevails over a-recoil 3) initial activity of ${ }^{228} \mathrm{Ra}$ and ${ }^{226} \mathrm{Ra}$ cannot be neglected/ a-recoil prevails over dissolution.

Initial activity of ${ }^{228}$ Ra and ${ }^{226}$ Ra can be neglected / a-recoil prevails over dissolution $(\Gamma>>\Delta)$ 
${ }^{228} \mathrm{Ra}_{w(0)}$ and ${ }^{226} \mathrm{Ra}_{w(0)}=0 ; \Delta_{2208} \Gamma_{228}=\Gamma_{228}$ and $\Delta_{228}$ $\Gamma_{226}=\Gamma_{226}$.

Remembering that $\Gamma_{228}=f_{a 228}, \lambda_{228}$ and $\Gamma_{226}=f_{a 226} \cdot \lambda_{226}$ Eq. 19 becomes:

$$
\frac{\left(\frac{{ }^{228} \mathrm{Ra}_{w}}{{ }^{226} \mathrm{Ra}_{w}}\right)}{\left(\frac{{ }^{228} \mathrm{Ra}_{r}}{{ }^{226} \mathrm{Ra}_{r}}\right)}=\frac{f_{\alpha 228}}{f_{\alpha 226}} \cdot \frac{\left(1-\mathrm{e}^{-\lambda_{228} \cdot \mathrm{R}_{R a-228} \cdot \mathrm{T}}\right)}{\left(1-\mathrm{e}^{-\lambda_{226} \cdot \mathrm{R}_{R a-228} \cdot \mathrm{T}}\right)}
$$

The $f_{a}$ of a Ra isotope $i$ depends on its recoil range in the particle, ${ }^{i} \mathrm{R}_{\mathrm{c}}$ (see Eq. 35-36) which in turn can be calculated by the following equation [99]:

${ }^{\text {i }} R_{c}=\left(M_{1}+M_{2}\right) \cdot\left(M_{2} \cdot E_{M 1} \cdot K\right) \cdot \frac{\left(Z_{1}^{2 / 3}+Z_{2}^{2 / 3}\right)^{1 / 2}}{\left(M_{1} \cdot Z_{1} \cdot Z_{2}\right)}$

where $E_{M 1}$ is the initial recoil energy of a nucleus of mass $M_{1}$ and atomic number $Z_{1}$, moving in an absorber of average mass $M_{2}$ and average atomic number $Z_{2}, K$ is a constant value (6.02), ${ }^{i} R_{c}$ is the recoil range in the absorber given in $n m$ and $E_{M 1}$ is given in keV.

In general $f_{\alpha i} \backslash f_{\alpha i^{\prime}}={ }^{i} \mathrm{R}_{\mathrm{c}} \backslash{ }^{i \prime} \mathrm{R}_{\mathrm{c}} \approx \mathrm{E}_{\mathrm{Mi}} / \mathrm{E}_{\mathrm{Mi}^{\prime}} \approx \mathrm{Q}_{\alpha i} \backslash \mathrm{Q}_{\alpha i^{\prime}}$

where $\mathrm{Q}_{a}$ is the a-decay energy of thorium parent.

Since $\mathrm{Q}_{a \mathrm{Th}-228} / \mathrm{Q}_{a-\mathrm{Th}-230}=1.15 \mathrm{Eq} .41$ can be written as:

$\frac{\left(\frac{{ }^{228} \mathrm{Ra}_{w}}{{ }^{226} \mathrm{Ra}_{w}}\right)}{\left(\frac{{ }^{228} \mathrm{Ra}_{r}}{{ }^{226} \mathrm{Ra}_{r}}\right)}=1.15 \cdot \frac{\left(1-\mathrm{e}^{-\lambda_{228} \cdot \mathrm{R}_{R a-228} \cdot \mathrm{T}}\right)}{\left(1-\mathrm{e}^{-\lambda_{226} \cdot \mathrm{R}_{\mathrm{Ra}-228} \cdot \mathrm{T}}\right)}$
$\mathrm{R}_{R a-228}=\mathrm{R}_{R a-226}=1.75 \cdot \mathrm{R}_{R a-224}$

Initial activity of ${ }^{228} \mathrm{Ra}$ and ${ }^{226} \mathrm{Ra}$ can be neglected/ dissolution prevails over a-recoil $(\Delta>>\Gamma)$

${ }^{228} \mathrm{Ra}_{w(0)}$ and ${ }^{226} \mathrm{Ra}_{w(0)}=0 ; \Delta_{228}=\Gamma_{228}=\Delta_{228}$ and $\Delta_{226}$ $\Gamma_{226}=\Delta_{228}$.

Equation (17) becomes:

$\frac{\left(\frac{{ }^{228} \mathrm{Ra}_{w}}{{ }^{226} \mathrm{Ra}_{w}}\right)}{\left(\frac{{ }^{228} \mathrm{Ra}_{r}}{{ }^{226} \mathrm{Ra}_{r}}\right)}=\frac{\lambda_{226} \cdot \Delta_{228}}{\lambda_{228} \cdot \Delta_{226}} \cdot \frac{\left(1-\mathrm{e}^{-\lambda_{228} \cdot \mathrm{R}_{R a-228} \cdot \mathrm{T}}\right)}{\left(1-\mathrm{e}^{-\lambda_{226} \cdot \mathrm{R}_{R a-228} \cdot \mathrm{T}}\right)}$

We can assume to fall back into this case only if the left member of Eq. 46 is $\geq\left(\lambda_{226} / \lambda_{228}\right)$.

In case of congruent dissolution, $\Delta_{228}=\Delta_{226}$, therefore:

$\frac{\left(\frac{{ }^{228} \mathrm{Ra}_{\mathrm{w}}}{{ }^{226} \mathrm{Ra}_{w}}\right)}{\left(\frac{{ }^{228} \mathrm{Ra}_{r}}{{ }^{226} \mathrm{Ra}_{r}}\right)}=\frac{\lambda_{226}}{\lambda_{228}} \cdot \frac{\left(1-\mathrm{e}^{-\lambda_{228} \cdot \mathrm{R}_{R a-228} \cdot \mathrm{T}}\right)}{\left(1-\mathrm{e}^{-\lambda_{226} \cdot \mathrm{R}_{R a-228} \cdot \mathrm{T}}\right)}$

If $\Delta_{228} \neq \Delta_{226}$ dissolution rates can be however estimated by literature values [2]. Also in this case, the equation can be solved for T, measuring R by NAPL method and applying Eq. 46

Initial activity of ${ }^{228} \mathrm{Ra}$ and ${ }^{226} \mathrm{Ra}$ cannot be neglected - a-recoil prevails over dissolution $(\Gamma>>\Delta)$

If initial activity of ${ }^{226} \mathrm{Ra}$ and ${ }^{228} \mathrm{Ra}$ cannot be neglected and $\Delta_{228}+\Gamma_{228}=\Gamma_{228} ; \Delta_{226}+\Gamma_{226}=\Gamma_{226}$, then (Eq. 1):

${ }^{228} \mathrm{Ra}_{w}=\left[\frac{\left(\Gamma_{228}\right)}{\left(\lambda_{228} \cdot \mathrm{R}_{R a-228}\right)}\right] \cdot\left(1-\mathrm{e}^{-\lambda_{228} \cdot \mathrm{R}_{R a-228} \cdot \mathrm{T}}\right) \cdot\left({ }^{228} \mathrm{Ra}_{r} \cdot \frac{\rho_{r}}{\Phi}\right)+{ }^{228} \mathrm{Ra}_{w(0)} \cdot\left(\mathrm{e}^{-\lambda_{228} \cdot \mathrm{R}_{R a-228} \cdot \mathrm{T}}\right)$

${ }^{226} \mathrm{Ra}_{w}=\left[\frac{\left(\Gamma_{226}\right)}{\left(\lambda_{226} \cdot \mathrm{R}_{R a-226}\right)}\right] \cdot\left(1-\mathrm{e}^{-\lambda_{226} \cdot \mathrm{R}_{R a-226} \cdot \mathrm{T}}\right) \cdot\left({ }^{226} \mathrm{Ra}_{r} \cdot \frac{\rho_{r}}{\Phi}\right)+{ }^{226} \mathrm{Ra}_{w(0)} \cdot\left(\mathrm{e}^{-\lambda_{226} \cdot \mathrm{R}_{R a-226} \cdot \mathrm{T}}\right)$

We can assume to fall within this case only if the left member of Eq. 44 is $\geq 1$. The equation can be solved for $T$, measuring $\mathrm{R}_{\mathrm{Ra}-224}$ by NAPL method and remembering that:
Since $\Gamma_{228} / \lambda_{228}=f_{a 228} ; \Gamma_{226} / \lambda_{226}=f_{a 226}$ and $f_{a 228}=f_{a 224} \cdot{ }^{228} \mathrm{R}_{c} /{ }^{224} \mathrm{R}_{\mathrm{c}}=f_{a 224} \cdot 0.74$. $f_{a 226}=f_{a 224} \cdot{ }^{226} \mathrm{R}_{\mathrm{c}} /{ }^{224} \mathrm{R}_{\mathrm{c}}=f_{a 224} \cdot 0.86$. where $R_{c}$ is the recoil range in the particle, then:

${ }^{228} \mathrm{Ra}_{w}=\left[\frac{f_{\alpha 224} \cdot 0.74}{\mathrm{R}_{R a-228}}\right] \cdot\left(1-\mathrm{e}^{-\lambda_{228} \cdot \mathrm{R}_{R a-228} \cdot \mathrm{T}}\right) \cdot\left({ }^{228} \mathrm{Ra}_{r} \cdot \frac{\rho_{r}}{\Phi}\right)+{ }^{228} \mathrm{Ra}_{w(0)} \cdot\left(\mathrm{e}^{-\lambda_{228} \cdot \mathrm{R}_{R a-228} \cdot \mathrm{T}}\right)$ 
${ }^{226} \mathrm{Ra}_{w}=\left[\frac{f_{\alpha 224} \cdot 0.86}{\mathrm{R}_{R a-226}}\right] \cdot\left(1-\mathrm{e}^{-\lambda_{226} \cdot \mathrm{R}_{R a-226} \cdot \mathrm{T}}\right) \cdot\left({ }^{226} \mathrm{Ra}_{r} \cdot \frac{\rho_{r}}{\Phi}\right)+{ }^{226} \mathrm{Ra}_{w(0)} \cdot\left(\mathrm{e}^{-\lambda_{226} \cdot \mathrm{R}_{R a-226} \cdot \mathrm{T}}\right)$

By manipulating Eqs. 50-51:

$\left[\frac{{ }^{228} \mathrm{Ra}_{w}-\left({ }^{228} \mathrm{Ra}_{w(0)} \cdot \mathrm{e}^{-\lambda_{228} \cdot \mathrm{R}_{R a-228} \cdot \mathrm{T}}\right)}{{ }^{226} \mathrm{Ra}_{w}-\left({ }^{226} \mathrm{Ra}_{w(0)} \cdot \mathrm{e}^{-\lambda_{226} \cdot \mathrm{R}_{R a-228} \cdot \mathrm{T}}\right)}\right]=\frac{0.74}{0.86} \cdot \frac{{ }^{228} \mathrm{Ra}_{r}}{{ }^{226} \mathrm{Ra}_{r}} \cdot \frac{\left(1-\mathrm{e}^{-\lambda_{228} \cdot \mathrm{R}_{R a-228} \cdot \mathrm{T}}\right)}{\left(1-\mathrm{e}^{-\lambda_{226} \cdot \mathrm{R}_{R a-228} \cdot \mathrm{T}}\right)}$

By knowing ${ }^{228} \mathrm{Ra}_{w(0)},{ }^{226} \mathrm{Ra}_{\mathrm{w}(0)}$ and measuring $\mathrm{R}_{\mathrm{Ra}}$ by NAPL method, Eq. 52 can be solved for T.

\section{References}

1. Suckow A (2014) The age of groundwater-definitions, models and why we do not need this term. Appl Geochem 50:222-230

2. Rajaomahefasoa RE, Voltaggio M, Rakotomandrindra PF, Ratsimbazafya JB, Spadoni M, Rakoto HA (2019) Radium isotopes for groundwater age and sustainability in the highland of Antananarivo, Madagascar. J Afr Earth Sci 156:94-107

3. Luo X, Jiao JJ (2019) Unraveling controlling factors of concentration discharge relationships in a fractured aquifer dominant spring-shed. Evidence from mean transit time and radium reactive transport model. J Hydrol 571:528-544

4. Battaglia A, Ceccarelli A, Ridolfi A, Froehlich K, Panichi C (1992) Radium isotopes in geothermal fluids in central Italy. IAEA proceeding series. In: International symposium on isotope techniques in water resources development, Vienna, INIS, .pp 363-383

5. Froehlich K, Battaglia A, Ceccarelli A, Ridolfi A, Panichi C (1995) Radium isotopes contribution to geothermal exploration in Central Italy. Section 4: Exploration and Conceptual Modeling World Geothermal Congress, Latera, Italy, pp 1059-1064. https ://www.geothermal-energy.org/pdf/IGAstandard/WGC/1995/2Froehlich.pdf. Accessed 23 Jul 2019

6. Kiro Y,Yechieli Y, Voss Cl, Starinsky A, Weinstein Y (2012) Modeling radium distribution in coastal aquifers during sea level changes : The Dead Sea case.Geochimica et Cosmochimica Acta. 2012; 88:237-254.

7. Kiro Y, Weinstein Y, Starinsky A, Yechieli Y (2013) Groundwater ages and reaction rates during seawater circulation in the Dead Sea aquifer. Geochim Cosmochim Acta 122:17-35

8. Voltaggio M, Spadoni M, Sacchi E, Sanam R, Pujari PR, Labhasetwar PK (2015) Assessment of groundwater pollution from ash ponds using stable and unstable isotopes around the Koradi and Khaperkheda thermal power plants (Maharashtra, India). Sci Total Environ 518-519:616-625

9. Mojid MA, Vereecken $\mathrm{H}$ (2005) On the physical meaning of retardation factor and velocity of a nonlinearly sorbing solute. J Hydrol 302:127-136

10. Krishnaswami S, Graustein WS, Turekian KK, Dowd JF (1982) Radium, thorium and radioactive lead isotopes in groundwaters: application to the in situ determination of adsorption-desorption rate constants and retardation factors. Water Resour Res 18:1633-1675

11. Suzuki K (1987) Discordant distribution of $U$ and $\mathrm{Pb}$ in zircon of Naegi granite: a possible indication of Rn migration through radiation damage. Geochem J 21:173-182
12. Sakoda A, Ishimori $Y$ (2017) Mechanisms and modeling approaches of radon emanation for natural materials. Jpn J Health Phys 52:296-306

13. Maraziotis EA (1996) Effects of intraparticle porosity on the radon emanation coefficient. Environ Sci Technol 30:2441-2448

14. Baskaran M (2016) Mechanism of radon emanation and longterm radon fluxes studies. In: Radon: a tracer for geological, geophysical and geochemical studies. Springer, Cham, pp 37-62

15. Han A, Kong X, Qiao Y (2006) Pressure indued liquid infiltration in nanopores. J Appl Phys 100:014308-014310

16. Sun Y, Li P, Qiao Yu, Li Y (2014) Time-dependent gas-liquid interaction in molecular-sized nanopores. Sci Rep. https://doi. org/10.1038/srep06547

17. Rama MWS (1984) Mechanism of transport of U-Th series radioisotopes from solids into ground water. Geochim Cosmochim Acta 48:395-399

18. Krishnaswami S, Seidemann DE (1988) Comparative study of ${ }^{222} \mathrm{Rn},{ }^{40} \mathrm{Ar},{ }^{39} \mathrm{Ar}$ and ${ }^{37} \mathrm{Ar}$ leakage from rocks and minerals: Implications for the role of nanopores in gas transport through natural silicates. Geochim Cosmochim Acta 52:655-658

19. Morawska L, Phillips CR (1993) Dependence of the radon emanation coefficient on radium distribution and internal structure ef the material. Geochim Cosmochim Acta 57:1783-1197

20. Wood WW, Kraemer TF, Shapiro A (2004) Radon $\left({ }^{222} \mathrm{Rn}\right)$ in ground water of fractured rocks: a diffusion/ion exchange model. Ground Water 42:552-567

21. Reynolds BC, Wasserburg GJ, Baskaran M (2003) The transport of $\mathrm{U}$ - and Th-series nuclides in sandy confined aquifers. Geochim Cosmochim Acta 67(11):1955-1972

22. Semkow TM (1990) Recoil-emanation theory applied to radon release from mineral grains. Geochim Cosmochim Acta 54:425-440

23. Eyal Y, Fleisher RL (1985) Preferential leaching and the age of radiation damage from alpha decay in minerals. Geochim Cosmochim Acta 49:1155-1164

24. Semkow TM (1991) Fractal model of radon emanation from solids. Phys Rev Lett 66:3012-3015

25. Özgümüs A, Barillon R, Chambaudet A, Groetz JE (1999) Theoretical study of the radon emanation coefficient for granular material : influence of packing and water. In: Radon in living environment 19-23 April 1999, Athens, Greece, pp 1251-1258

26. Barillon R, Özgümüs $A$, Chambaudet $A$ (2005) Direct recoil radon emanation from crystalline phase Influence of moisture content. Geochim Cosmochim Acta 69:2735-2744

27. Fleisher $\mathrm{R}$ (1983) Theory of alpha recoil effects on radon release and isotopic disequilibrium. Geochimica Cosmochimica Acta 47:779-784

28. Barbieri M, Voltaggio M (1998) Applications of Sr isotopes and $\mathrm{U}$-series radionuclides to the hydrogeology of Sangemini area Terni, central Italy. Mineral Petrogr Acta 41:119-126

29. Sturchio NC, Banner JL, Binz CM, Heraty LB, Musgrove M (2001) Radium geochemistry of groundwaters in Paleozoic carbonate aquifers, midcontinent, USA. Appl Geochem 16:109-122 
30. Tricca A, Wasserburg GJ, Porcelli D, Baskaran M (2001) The transport of $\mathrm{U}$ - and Th-series nuclides in a sandy unconfined aquifer. Geochim Cosmochim Acta 65:1187-1210

31. Ohta T, Mahara Y, Miyakawa K, Kubota T, Tagami K, Sato J (2012) ${ }^{228} \mathrm{Ra} /{ }^{226} \mathrm{Ra}$ activity ratio in groundwater around Mount Fuji, Japan. EPJ Web of Conferences 3(24):03003

32. Vinson DS, Vengosh A, Hirschfeld D, Dwyer GS (2009) Relationships between radium and radon occurrence and hydrochemistry in fresh groundwater from fractured crystalline rocks, North Carolina (USA). Chem Geol 260:159-171

33. Beck AJ, Cochran MA (2013) Contros on solid-solutions partitioning of radium in saturated marine sands. Mar Chem 156:38-48

34. Kiro Y, Weinstein Y, Starinsky A, Yechieli Y (2015) Application of radon and radium isotopes to groundwater flow dynamics: an example from the Dead Sea. Chem Geol 2015(411):155-171

35. Karen LK, Garcia-Solsona E, Garcia-Orellana J, Boehm AB, Paytan $A$ (2011) Using radium isotopes to characterize water ages and coastal mixing rates: A sensitivity analysis. Limnol Oceanogr Methods 9:380-395

36. Finnegan DL, Bryant EA (1987) Methods for obtaining sorption data from uranium-series disequilibria. Los Alamos National Laboratory. LA-1162-MS-INIS 19:1-21. https://inis.iaea.org/ collection/NCLCollectionStore/ Public/19/059/19059741.pdf. Accessed 23 Jul 2019

37. Krishnaswami S, Bhushan R, Baskaran M (1991) Radium Isotopes and ${ }^{222} \mathrm{Rn}$ in Shallow Brines, Kharaghoda (India). Chem Geol (Isotope Geosci Sect) 87:125-136

38. Wainipee W, Cuadros J, Sephton MA, Unsworth C, Gill MG, Strekopytov S, Weiss DJ (2013) The effects of oil on As(V) adsorption on illite, kaolinite, montmorillonite and chlorite. Geochim Cosmochim Acta 121:487-502

39. Schwartz N, Huisman JA, Furman A (2012) The effect of NAPL on the electrical properties of unsaturated porous media. Geophys J Int 188:1007-1011

40. Farajzadeh R, Guo H, van Winden J, Bruining J (2017) Cation exchange in the presence of oil in porous media. Earth Space Chem 1:101-112

41. Michael H, Charette MA, Harvey CF (2011) Patterns and variability of groundwater flow and radium activity at the coast: a case study from Waquoit Bay. Massachusetts Marine Chem 127:100-114

42. Capelli G, Mazza R, Taviani S (2008) Le acque sotterranee della città di Roma. Memorie Descrittive della Carta Geologica d'Italia 80:241-245

43. Dickie JM (2010) Mineralogical and geochemical indicators of subaerial weathering in the pozzolane rosse ignimbrite (Alban Hills Volcanic District, Italy). Thesis, Georgia State University, 2010. https://scholarworks.gsu.edu/geosciences_theses/23. Accessed 23 Jul 2019

44. Freda C, Gaeta M, Giaccio B, Marra F, Palladino DM, Scarlato P, Sottili $\mathrm{G}$ (2011) $\mathrm{CO}_{2}$-driven large mafic explosive eruptions: the Pozzolane Rosse case study from the Colli Albani Volcanic District (Italy). Bull Volcanol 73:241-256

45. Voltaggio M, Spadoni, (2013) Determination of ${ }^{222} \mathrm{Rn}$ in water by absorption in polydimethylsiloxane mixed with activated carbon and gamma-ray spectrometry: An example application in the radon budget of paterno submerged sinkhole (central Italy). Appl Geochem 34:65-74

46. Mandujano-García CD, Sosa M, Vallejo MA, Mantero J, Vioque I, Manjón G, García-Tenorio R (2019) Radioactive content of lantern gas mantles used in night food stalls and camping. J Rad Nucl Appl 3:127-134

47. Šebesta F, Havlík B (1975) Synergistic extraction of radium using 2-thenoyltrifluoroacetone and tributyl phosphate or trioctylphosphine oxide. J Radioanal Chem 24:337-343
48. Moore WS, Reid DF (1973) Extraction of radium from natural waters using manganese-impregnated acrylic fibers. J Geophys Res 78:8880-8886

49. Nagar MS, Abdou AA, Ghazala RAS (2018) Removal of radium from uranium effluent by manganese oxide coated modified bentonite (Mn-NaB). Mediterranean J Chem 7:105-114

50. Patterer MS, Medicia F, Peluso MA, Sambeth JE (2018) Lead adsorption from aqueous solution using manganese oxides recovered from spent alkaline and $\mathrm{Zn} / \mathrm{C}$ batteries. In: Cisap8 international conference on safety \& environment in process \& power industry. https://www.aidic.it/cisap8/papers/9patterer. pdf. Accessed $23 \mathrm{Jul} 2019$

51. Thien SJ, Graveel JG (1996) Laboratory manual for soil science: agricultural \& environmental principles 1996. McGraw-Hill Higher Education, Technology \& Engineering

52. Liu Y, Yang W, Zhang P, Zhang J (2018) Nitric acid-treated birnessite-type $\mathrm{MnO}_{2}$ : an efficient and hydrophobic material for humid ozone decomposition. Appl Surf Sci 442:640-649

53. Jurado-Vargas $M$, Oliguín MT, Erdóñez-Regil E, Miménez-Reyes $M$ (1997) lon exchange of radium and barium in zeolites. J Radioanal Nucl Chem 218:153-156

54. Frenvik JO, Kristensen S, Ryan OB (2016) Development of separation technology for the removal of radium-223 from decayed thorium-227 in drug formulations. Material screening and method development. Drug Dev Ind Pharm 42:1215-1224

55. Bozzano F, Marcoccia S, Barbieri M (1999) The role of calcium carbonate in the geomechanical behaviour of Pliocene lacustrine deposits. Q J Eng Geol 32:271-289

56. Baldanza A, Bizzarri R, Di Matteo L, Lezzerini M, Mencaroni L, Pagnotta S, Raneri S, Vinti G (2018) New integrated data from clay lacustrine deposits of the Dunarobba area (Umbria, central Italy). Alpine Mediterranean Quater 31:87-104

57. Barbieri M, Voltaggio M (1997) Hydraulic permeability and natural radionuclides migration in clayey lacustrine sediments from the Plio-pleistocenic Tiberin Lake, central Italy, in relation to the waste disposal. In:Marinos, Koukis, Tsiambaos, Stournaras (eds) Engineering geology and the environment. Proceedings International symposium on engineering geology and the environment/laeg/Athens/Greece/23-27 June 1997. Rotterdam, Brookfield, pp 1587-1592

58. Chao JH, Niu H, Chiu CY, Lin C (2007) A potential dating technique using ${ }^{228} \mathrm{Th} /{ }^{228} \mathrm{Ra}$ for tracing the chronosequence of elemental concentration in plants. Appl Radiat Isot 65:641-648

59. Luo S, Ku TL, Robacl R, Murrell M, McLing TL (2000) In-situ radionuclide transport and preferential groundwater flows at INEEL (Idaho): Decay series disequilibrium studies. Geochim Cosmochim Acta 64:867-881

60. Cinelli G, Capaccioni B, Hernández-Ceballos MA, Mostacci D, Perghem A, Tositti L (2015) Radiological risk from thoron, a case study: the particularly radon-prone area of Bolsena, and the lesson learned. Radiat Phys Chem 116:381-385

61. Lombardi G, Mattias P (1979) Petrology and mineralogy of the kaolin and alunite mineralization of Latium (Italy). Geol Romana 18:157-214

62. Vignaroli G, Aldega L, Balsamo F, Billi A, De Benedetti AA, De Filippis L, Rossetti $F$ (2014) A way to hydrothermal paroxysm, Colli Albani volcano, Italy. Geol Soc Am Bull B31139-1

63. Giordano G, The CARG Team (2010) Stratigraphy, volcano tectonics and evolution of the Colli Albani volcanic field. In: The Colli Albani Volcano. Geological Society, London, pp43-98

64. Schmidt S, Reyss JL (1996) Radium as internal tracer of Mediterranean Outflow Water. J Geophys Res 101:3589-3596

65. Jackson M, Deocampo D, Marra F, Scheetz B (2010) Mid-Pleistocene pozzolanic volcanic ash in ancient Roman concretes. Geoarchaeology 25:36-74 
66. Benes $\mathrm{P}$ (1982) Physico-chemical forms and migration in continental waters of radium from uranium mining and milling. In: Environmental migration of long-lived radionuclides. Proceeding series IAEA-SM-257, International Atomic Energy Agency, Vienna, Austria, pp 3-23

67. Nathwani JS, Phillips CR (1979) Adsorption of ${ }^{226}$ Ra by soils in the presence of $\mathrm{Ca}^{2+}$ ions. Specific adsorption (II). Chemosphere 5:293-299

68. Pan G, Liss PS, Krom M (1999) Particle concentration effect and adsorption irreversibility. Colloids Surfaces A: Physicochem Eng Aspects 151:127-133

69. Qin YW, Pan G, Zhang MM, Li XL (2004) Adsorption of zinc on manganite $(\gamma-\mathrm{MnOOH})$ : particle concentration effect and adsorption reversibility. J Environ Sci 16:627-630

70. Schmidt WF, Volykhin KF, Khrapak AG, Illenberger E (1999) Structure and mobility of positive and negative ions in non-polar liquids. J Electrostat 47:83-95

71. Smith G, Eastoe J (2013) Controlling colloid charge in nonpolar liquids with surfactants. Phys Chem Chem Phys 15:424-439

72. Dukhin AS, Goetz PJ (2010) Characterization of liquids, nanoand microparticulates and porous bodies using ultrasound. Studies in Interface Science. Elsevier, Amsterdam

73. Gray E, Lewis TJ (1969) The effect of liquid motion on ion mobility in hexane. J Phys D Appl Phys 2:93-100

74. Davies O, Goldsmith PL (1972) Statistical method in research and production. Oliver and Boyd, Edinburgh

75. Thijssen L, Schaart DR, de Vries D, Morgenstern A, Bruchertseifer F, Denkova AG (2012) Polymersomes as nano-carriers to retain harmful recoil nuclides in alpha radionuclide therapy; a feasibility study. Radiochim Acta 100:473-480

76. Hernández WY, Centenoa MA, Romero-Sarria F, Ivanovaa S, Montes M, Odriozola JA (2010) Modified cryptomelane-type manganese dioxide nanomaterials for preferential oxidation of CO in the presence of hydrogen. Catal Today 157:160-165

77. Srither SR, Karthik A, Selvam M, Saminathan K, Rajendran V, Kaler KVIS (2014) Nano-sized $\mathrm{MnO}_{2}$ particles produced by spray pyrolysis for a $\mathrm{Zn} / \mathrm{MnO} 2$ primary cell: comparative discharge performance studies with their bulkcounterpart. RSC Adv 4:42129-42136

78. Zhang J, Lu S, Li J, Zhang P, Xue H, Zhao X, Xie L (2017) Adsorption properties of hydrocarbons ( $n$-decane, methyl cyclohexane and Toluene) on clay minerals: an experimental study. Energies 10:586. https://doi.org/10.3390/en10101586

79. Santamarina JC, Fam M (1997) Dielectric permittivity of soils mixed with organic and inorganic fluids $(0.02 \mathrm{GHz}$ to $1.30 \mathrm{GHz})$. J Environ Eng Geophys 2:37-52

80. Mushidi J, Anderson C (2017) Surface chemistry and flotation behavior of monazite, apatite, ilmenite, quartz, rutile, and zircon with octanohydroxamic acid. J Sustain Metall 3:62-72

81. Clavier N, Podor R, Dacheux N (2011) Crystal chemistry of the monazite structure. J Eur Ceram Soc 31:941-976

82. Zhang W, Honaker RQ, Groppo JG (2017) Flotation of monazite in the presence of calcite part I: Calcium ion effects on the adsorption of hydroxamic acid. Miner Eng 100:40-48

83. Briganti A, Voltaggio M, Soligo M, Tuccimei P (2018) Assessing the age of a NAPL-spill by Radium isotopes. In: Book of the abstracts. 7th international RaRn workshop 2nd-6th July. Alfred Wegener Institute, Delmenhorst, pp 41

84. Smeets RMM, Keyser UF, Wu MY, Dekker NH, Dekker C (2006) nanobubbles in solid-state nanopores. Phys Rev Lett 97:088101-088104
85. de la Llave E, Molinero V, Scherlis DA (2010) Water filling of hydrophilic nanopores. J Chem Phys 133(034513):1-10

86. Sugita F, Gillham RW (1995) Pore scale variation in retardation factor as a cause of non ideal reactive breakthrough curves 1. Conceptual model and its evaluation. Water Resour Res 31:103-112

87. Ozgumus T, Mobedi M, Ozkol U (2014) Determination of Kozeny constant based on porosity and pore to throat size ratio in porous medium with rectangular rods. Eng Appl Comput Fluid Mech 8:308-318

88. Pelizza S, Peila D, Sorge R, Cignitti F (2012) Back-fill grout with two component mix in EPB tunneling to minimizesurface settlements: Rome metro-line C case history. In: Viggiani G (ed) Geotechnical aspects of underground construction in soft ground. Taylor \& Francis Group, London, pp 291-299

89. Furnari S, Martarelli L, Moroni M (2010) Hydrogeological model in a test area of the Alban Hills, Rome, Central Italy, In: Global groundwater resources and management. Scientific Publishers, Jodhpur, pp 185-205

90. Colombier M, Wadsworth FB, Gurioli L, Scheu B (2017) The evolution of pore connectivity in volcanic rocks. Earth Planet Sci Lett 462:99-109

91. Weissmann GS, Zhang Y, LaBolle EM, Fogg GE (2002) Dispersion of groundwater age in an alluvial aquifer system. Water Resour Res 38:1-13. https://doi.org/10.1029/2001WR000907

92. Cook PG, Bohlke JK (2000) Determining timescales for groundwater flow and solute transport. In: Cook PG, Herczeg AL (eds) Environmental tracers in subsurface hydrology. Kluwer, pp 1-30

93. Chen $\mathrm{MH}$, Cardoso SSS (2000) The mixing of liquids by a plume of low-Reynolds number bubbles. Chem Eng Sci 55:2585-2594

94. La Vigna F, Mazza R, Amanti M, Di Salvo C, Petitta M, Pizzino L, Pietrosante A, Martarelli L, Bonfà I, Cinti D, Ciotoli G, Conte G, Del Bon A, Dimasi M, Falcetti S, Gafà R, Lacchini A, Alessandro, Mancini M, Martelli S, Ciotoli F (2015) Carta Idrogeologica di Roma Hydrogeological Map of Rome 2015. https://www.resea rchgate.net/publication/281966009_Carta_Idrogeologica_di_ Roma_Hydrogeological_Map_of_Rome. Accessed 23 Jul 2019.

95. Jia G, Torri G (2007) Estimation of radiation doses to members of the public in Italy from intakes of some important naturally occurring radionuclides in drinking water ${ }^{238} \mathrm{U},{ }^{234} \mathrm{U},{ }^{235} \mathrm{U},{ }^{226} \mathrm{Ra}$, ${ }^{228} \mathrm{Ra},{ }^{224} \mathrm{Ra}$ and ${ }^{210} \mathrm{Po}$ ). Appl Radiat Isot 65:849-857

96. Cherniak DJ (2002) Ba diffusion in feldspar. Geochim Cosmochim Acta 66:1641-1650

97. Copenhaver SA, Krishnaswami S, Turekian KK, Shaw H (1992) ${ }^{238} \mathrm{U}$ and ${ }^{232} \mathrm{Th}$ series nuclides in ground water from the J-13 well at the Nevada test site; implications for the ion retardation. Geophys Res Lett 19:1383-1386

98. Copenhaver SA, Krishnaswami S, Turekian KK, Epler N, Cochran JK (1993) Retardation of ${ }^{238} \mathrm{U}$ and ${ }^{232} \mathrm{Th}$ decay chain radionuclides in Long Island and Connecticut aquifers. Geochim Cosmochim Acta 57:597-603

99. Voltaggio M, Palmisano M, Raponi A, Voltaggio S (2001) Implantation of recoiling radionuclides of $U$ and Th radioactiveseries applied to estimation of surficial erosion of $\mathrm{CaCO} 3$ materials. Appl Geochem 16:835-848

Publisher's Note Springer Nature remains neutral with regard to jurisdictional claims in published maps and institutional affiliations. 